\title{
Synthesis and structural characterization of some molybdenum carbonyl nitrosyl complexes of diethyldithiocarbamate
}

\author{
Kom-Bei Shiu ${ }^{a, *}$, Sheng-Ting Lin ${ }^{a}$, Shie-Ming Peng ${ }^{b}$, Ming-Chu Cheng ${ }^{b}$ \\ "Department of Chemistry, National Cheng Kung University, Tainan 701, Taiwan \\ ${ }^{\mathrm{b}}$ Department of Chemistry, National Taiwan University, Taipei 107, Taiwan
}

Received 27 May 1994; revised 26 July 1994

\begin{abstract}
Ten complexes, $\mathrm{M}^{\prime}\left[\mathrm{Mo}\left(\mathrm{Et}_{2} \mathrm{NCS}_{2}\right)(\mathrm{CO})_{2}(\mathrm{NO}) \mathrm{X}\right]\left(\mathrm{M}^{++}=\mathrm{PPN}^{+}\right.$or $\mathrm{Et}_{4} \mathrm{~N}^{+} ; \mathrm{X}^{-}=\mathrm{NCS}^{-}(1), \mathrm{NO}_{3}^{-}$(2), $\mathrm{N}_{3}^{-}(3), \mathrm{Br}^{-}$(4) or $\left.\mathrm{Cl}^{-}(5)\right)$ and $\left[\mathrm{Mo}\left(\mathrm{Et}_{2} \mathrm{NCS}_{2}\right)(\mathrm{CO})(\mathrm{NO}) \mathrm{L}_{2}\right]\left(\mathrm{L}_{2}=\right.$ py and $\mathrm{CO}(6)$; dppe $\left.(7) ; 2 \mathrm{PPh}_{3}(8) ; 2 \mathrm{PMe}_{3}(9) ; 2 \mathrm{P}(\mathrm{OMe})_{3}(10)\right)$, have been prepared. Crystal structures of $1,3,4,6,7,8$ and 10 were determined from single crystal X-ray data. The structures show clearly that the stronger electron-withdrawing $\mathrm{NO}$, compared with $\mathrm{CO}$, prefers the location trans to the better electron donor such as $\mathrm{NCS}^{-}$in $1, \mathrm{~N}_{3}^{-}$in 3 and $\mathrm{Br}^{-}$in 4 , unless some other apparent counteracting factors are also involved. The two bulky phosphine ligands are as expected trans to each other in 8 and 10 and a chelate dppe is found in 7 . Although the structure of 6 has NO trans to py, the spectral data of 6 (and 7), both in solution and in the solid state, reveal the presence of two different structures. Crystal data: 1 , space group $P 21 / n, a=9.879(2), b=30.302(3), c=14.858(4) \AA, \beta=97.00(2)^{\circ}, Z=4$; 3, space group $P 2_{1} / c, a=16.806(5), b=16.128(3), c=18.025(4) \AA, \beta=116.94(2)^{\circ}, Z=4 ; 4$, space group Ia, $a=14.773(8)$, $b=8.982(2), c=17.748(5) \AA, \beta=96.49(5)^{\circ}, Z=4 ; 6$, space group $P 2_{1} / c, a=13.499(4), b=10.033(5), c=12.962(2) \AA, \beta=91.66(2)^{\circ}$, $Z=4 ; 7$, space group $P 2_{1} / n, a=11.634(3), b=19.881(4), c=15.225(4) \AA, \beta=111.10(2)^{\circ}, Z=4 ; 8$, space group $P 2_{1} / n, a=11.767(2)$, $b=18.851(4), c=19.436(4) \AA, \beta=107.12(2)^{\circ}, Z=4 ; 10$, space group $P 2 / n, a=8.732(2), b=23.98(3), c=11.303(3) \AA, \beta=97.66(2)^{\circ}$, $Z=4$.
\end{abstract}

Keywords: Crystal structures; Molybdenum complexes; Carbonyl complexes; Nitrosyl complexes; Dithiocarbamate complexes

\section{Introduction}

Following the discovery of the symmetrical and asymmetrical orientations of the allyl group with respect to the neutral pyrazole-derived tridentate ligand $(\mathrm{N}-\mathrm{N}-\mathrm{N})$ in cations, $\left[\mathrm{M}(\mathrm{N}-\mathrm{N}-\mathrm{N})(\mathrm{CO})_{2}\left(\eta^{3} \text {-allyl }\right)\right]^{+}(\mathrm{M}=\mathrm{Cr}$, Mo and $\mathrm{W})[1,2]$, we were interested in examining the structure predictability from the electronic and steric property of the associated ligands. In this paper we report some related results from the syntheses and structures of a number of six-coordinate complexes of molybdenum, $\left[\mathrm{Mo}\left(\mathrm{Et}_{2} \mathrm{NCS}_{2}\right)(\mathrm{NO})(\mathrm{CO}) \mathrm{L}_{2}\right]^{m^{-}}(m=0$ or 1; $\mathrm{L}_{2}=1,2$-bis(diphenylphosphino)ethane (dppe) or $\mathrm{L}=\mathrm{PPh}_{3}, \mathrm{PMe}_{3}, \mathrm{P}(\mathrm{OMe})_{3}, \mathrm{Br}^{-}, \mathrm{Cl}^{-}, \mathrm{N}_{3}{ }^{-}, \mathrm{NO}_{3}{ }^{-}, \mathrm{NCS}^{-}$ or pyridine (py)).

\footnotetext{
Dedicated to Professor F. Albert Cotton on the occasion of his 65th birthday and the graduation of his 100th Ph.D. student.

*Corresponding author.
}

\section{Experimental}

All manipulations were carried out under an atmosphere of prepurified nitrogen by using conventional Schlenk-tube techniques. Solvents were purified by distillation from an appropriate drying agent (ethers, paraffins and arenes from potassium with benzophenone as indicator; halocarbons and acetonitrile from $\mathrm{CaH}_{2}$ and alcohols from the corresponding alkoxide).

${ }^{1} \mathrm{H}$ and ${ }^{13} \mathrm{C}$ NMR spectra were recorded on a Bruker AM-200 $\left({ }^{1} \mathrm{H}, 200 \mathrm{MHz} ;{ }^{13} \mathrm{C}, 50 \mathrm{MHz}\right)$ or AMC-400 $\left({ }^{1} \mathrm{H}, 400 \mathrm{MHz}\right)$ NMR spectrometer and chemical shifts $(\delta \mathrm{ppm}, J \mathrm{~Hz})$ are positive downfield relative to internal $\mathrm{MeSi}_{4}$ (TMS) or the deuterated solvent (s, singlet; d, doublet; $\mathrm{m}$, multiplet; br, broad). ${ }^{31} \mathrm{P}$ NMR spectra were recorded on a Bruker AMC-400 $\left({ }^{31} \mathrm{P}, 162 \mathrm{MHz}\right)$ NMR spectrometer and the chemical shifts $(\delta \mathrm{ppm})$ are defined as positive downfield relative to external $85 \% \mathrm{H}_{3} \mathrm{PO}_{4}$. IR spectra were recorded by using a Hitachi 270-30 instrument (vs, very strong; s, strong; m, medium; w, weak; br, broad; sh, shoulder). Elemental 
analysis results were obtained by the staff of the Microanalytical Service of the Department of Chemistry, National Cheng Kung University. $\left[\mathrm{Mo}\left(\mathrm{Et}_{2} \mathrm{NCS}_{2}\right)_{-}\right.$ $\left.(\mathrm{CO})_{2}(\mathrm{NO})\right]_{2}$ was prepared by the published procedure [3].

\subsection{Synthesis of \\ $[\mathrm{PPN}]\left[\mathrm{Mo}\left(\mathrm{Et}_{2} \mathrm{NCS}_{2}\right)(\mathrm{CO})_{2}(\mathrm{NO})(\mathrm{NCS})\right]$}

To a $50 \mathrm{ml}$ round-bottom flask containing a magnetic bar, $0.099 \mathrm{~g}$ of $\left[\mathrm{Mo}\left(\mathrm{Et}_{2} \mathrm{NCS}_{2}\right)(\mathrm{CO})_{2}(\mathrm{NO})\right]_{2}(0.15 \mathrm{mmol})$ and $0.18 \mathrm{~g}$ of $\mathrm{PPN}^{+} \mathrm{SCN}^{-}(0.30 \mathrm{mmol})\left(\mathrm{PPN}^{+}=\right.$ bis(triphenylphosphino)iminium $(1+)$ cation) were added to $15 \mathrm{ml}$ of $\mathrm{CH}_{2} \mathrm{Cl}_{2}$. The resulting solution was stirred for $15 \mathrm{~min}$ at $28^{\circ} \mathrm{C}$ and its color changed from orange-red to orange-yellow. The solvent was then removed under vacuum. Recrystallization from $\mathrm{CH}_{2} \mathrm{Cl}_{2}$ / $\mathrm{MeOH}$ gave a yellow product $(0.24 \mathrm{~g}, 86 \%)$. Anal. Calc. for $\mathrm{C}_{44} \mathrm{H}_{40} \mathrm{MoN}_{4} \mathrm{O}_{3} \mathrm{P}_{2} \mathrm{~S}_{3}$ : C, 57.02; $\mathrm{H}, 4.35 ; \mathrm{N}, 6.04$. Found: C, 56.89; H, 4.35; N, 6.00\%. ${ }^{1} \mathrm{H}$ NMR $\left(23{ }^{\circ} \mathrm{C}\right.$, acetone- $\left.\mathrm{d}_{6}, 200 \mathrm{MHz}\right) ; \delta$, phenyl protons $7.70(\mathrm{~m}, 30 \mathrm{H})$; ethyl protons, $3.81(\mathrm{q}, 2 \mathrm{H}), 3.84(\mathrm{q}, 2 \mathrm{H}), 1.24(\mathrm{t}, 6 \mathrm{H}$, $\left.{ }^{3} J(\mathrm{H}, \mathrm{H})=7.1\right)$. IR $\left(\mathrm{KBr}, \mathrm{cm}^{-1}\right): \nu(\mathrm{CO}) 2012 \mathrm{~s}, 1926 \mathrm{~s}$; $\nu$ (NO) $1628 \mathrm{~s} ; \nu(\mathrm{CN})$ of $\mathrm{Et}_{2} \mathrm{NCS}_{2}^{-}, 1496 \mathrm{~s} ; \nu(\mathrm{CN})$ of $\mathrm{NCS}^{-}$, 2076s. IR $\left(\mathrm{CH}_{2} \mathrm{Cl}_{2}, \mathrm{~cm}^{-1}\right): \nu(\mathrm{CO}) 2016 \mathrm{~s}, 1924 \mathrm{~s}$; $\nu(\mathrm{NO}) 1626 \mathrm{~s} ; \nu(\mathrm{CN})$ of $\mathrm{Et}_{2} \mathrm{NCS}_{2}^{-}, 1492 \mathrm{~s} ; \nu(\mathrm{CN})$ of $\mathrm{SCN}^{-}, 2076 \mathrm{~s}$.

\subsection{Synthesis of [PPN][Mo(Et $\left.t_{2} N C S_{2}\right)(C O)_{2}(\mathrm{NO})\left(\eta^{1}-\right.$ $\left.\left.\mathrm{NO}_{3}\right)\right](2)$}

This yellow compound was prepared in a yield of $71 \%$ by a procedure similar to that used for 1 , using $\mathrm{PPN}^{+} \mathrm{NO}_{3}{ }^{-}$. Anal. Calc. for $\mathrm{C}_{43} \mathrm{H}_{40} \mathrm{MoN}_{4} \mathrm{O}_{6} \mathrm{P}_{2} \mathrm{~S}_{2}$ : C, 55.49; H, 4.33; N, 6.02. Found: C, 55.27; H, 4.33; N, $5.76 \%$. ${ }^{1} \mathrm{H}$ NMR $\left(23{ }^{\circ} \mathrm{C}\right.$, acetone- $\left.\mathrm{d}_{6}, 200 \mathrm{MHz}\right): \delta$, phenyl protons $7.70(\mathrm{~m}, 30 \mathrm{H})$; ethyl protons $3.85(\mathrm{q}$, $\left.2 \mathrm{H},{ }^{3} \mathrm{~J}(\mathrm{H}, \mathrm{H})=7.1\right), 3.80\left(\mathrm{q}, 2 \mathrm{H},{ }^{3} \mathrm{~J}(\mathrm{H}, \mathrm{H})=7.1\right), 1.23(\mathrm{t}$, $3 \mathrm{H}), 1.22(\mathrm{t}, 3 \mathrm{H})$. IR $\left(\mathrm{KBr}, \mathrm{cm}^{-1}\right): \nu(\mathrm{CO}) 2016 \mathrm{~s}, 1930 \mathrm{~s}$; $\nu$ (NO) $1626 \mathrm{~s} ; \nu(\mathrm{CN}) 1496 \mathrm{~s}$. IR $\left(\mathrm{CH}_{2} \mathrm{Cl}_{2}, \mathrm{~cm}^{-1}\right): \nu(\mathrm{CO})$ 2020s, 1926s; $\nu(\mathrm{NO}) 1616 \mathrm{~s} ; \nu(\mathrm{CN}) 1486 \mathrm{~s}$.

\subsection{Synthesis of [PPN][Mo $\left(\mathrm{Et}_{2} \mathrm{NCS}_{2}\right)(\mathrm{CO})_{2}(\mathrm{NO})\left(\eta^{I}-\right.$ $\left.\left.N_{3}\right)\right](3)$}

This yellow compound was prepared in a yield of $88 \%$ by a procedure similar to that used for 1 , using $\mathrm{PPN}^{+} \mathrm{N}_{3}{ }^{-}$. Anal. Calc. for $\mathrm{C}_{43} \mathrm{H}_{40} \mathrm{MoN}_{6} \mathrm{O}_{3} \mathrm{P}_{2} \mathrm{~S}_{2}: \mathrm{C}, 56.70$; $\mathrm{H}, 4.43 ; \mathrm{N}, 9.23$. Found: C, 56.48; H, 4.43; N, 8.97\%. ${ }^{1} \mathrm{H}$ NMR $\left(23{ }^{\circ} \mathrm{C}\right.$, acetone- $\left.\mathrm{d}_{6}, 200 \mathrm{MHz}\right): \delta$, phenyl protons $7.70(\mathrm{~m}, 30 \mathrm{H})$; ethyl protons $3.80(\mathrm{q}, 2 \mathrm{H}$, $\left.{ }^{3} J(\mathrm{H}, \mathrm{H})=7.1\right), 3.78\left(\mathrm{q}, 2 \mathrm{H},{ }^{3} J(\mathrm{H}, \mathrm{H})=7.1\right), 1.24(\mathrm{t}, 3 \mathrm{H})$, $1.23(\mathrm{t}, 3 \mathrm{H}) \cdot{ }^{13} \mathrm{C}\left\{{ }^{1} \mathrm{H}\right\}$ NMR $\left(23{ }^{\circ} \mathrm{C}, \mathrm{CDCl}_{3}, 50 \mathrm{MHz}\right)$ : $\delta$, carbonyls $223.52 ; \mathrm{CN}, 214.52$; phenyl carbons 133.91 , $133.88,132.24,132.18,132.07,131.95,131.88,129.77$, $129.71,129.58,129.44,129.40,128.00,125.85$; ethyl carbons 44.90, 12.38. IR $\left(\mathrm{KBr}, \mathrm{cm}^{-1}\right): \nu\left(\mathrm{N}_{3}\right) 2060 \mathrm{~s}$; $\nu(\mathrm{CO}) 2000 \mathrm{~s}, 1914 \mathrm{~s} ; \nu(\mathrm{NO}) 1596 \mathrm{~s} ; \nu(\mathrm{CN}) 1506$. IR $\left(\mathrm{CH}_{2} \mathrm{Cl}_{2}, \mathrm{~cm}^{-1}\right): \nu\left(\mathrm{N}_{3}\right) 2064 \mathrm{~s} ; \nu(\mathrm{CO}) 2008 \mathrm{~s}, 1920 \mathrm{~s}$; $\nu(\mathrm{NO}) 1608 \mathrm{~s} ; \nu(\mathrm{CN}) 1494 \mathrm{~s}$.

\subsection{Synthesis of $\left[E t_{4} N\right]\left[\mathrm{Mo}\left(\mathrm{Et}_{2} \mathrm{NCS}_{2}\right)(\mathrm{CO})_{2}(\mathrm{NO})(\mathrm{Br})\right]$} (4)

This yellow compound was prepared in a yield of $92 \%$ by a procedure similar to that used for 1 , using $\mathrm{Et}_{4} \mathrm{~N}^{+} \mathrm{Br}^{-}$. Anal. Calc. for $\mathrm{C}_{15} \mathrm{H}_{30} \mathrm{BrMoN}_{3} \mathrm{O}_{3} \mathrm{~S}_{2}: \mathrm{C}, 33.34$; $\mathrm{H}, 5.60 ; \mathrm{N}, 7.78$. Found: C, 33.35; H, 5.62; N, 7.76\%. ${ }^{1} \mathrm{H}$ NMR $\left(23^{\circ} \mathrm{C}\right.$, acetone- $\left.\mathrm{d}_{6}, 200 \mathrm{MHz}\right): \delta, \mathrm{Et}_{4} \mathrm{~N}^{+} 1.39$ $\left(\mathrm{tt}, 12 \mathrm{H},{ }^{3} J(\mathrm{H}, \mathrm{H})=7.25,{ }^{3} J(\mathrm{~N}, \mathrm{H})=1.86\right), 3.50(\mathrm{q}, 8 \mathrm{H})$; $\mathrm{Et}_{2} \mathrm{NCS}_{2}{ }^{-} 1.23\left(\mathrm{t}, 6 \mathrm{H},{ }^{3} \mathrm{~J}(\mathrm{H}, \mathrm{H})=7.1\right), 3.80(\mathrm{q}, 2 \mathrm{H}), 3.79$ (q, 2H). IR (KBr, cm $\left.{ }^{-1}\right): \nu(\mathrm{CO}) 2012 \mathrm{~s}, 1908 \mathrm{~s} ; \nu(\mathrm{NO})$ $1608 \mathrm{~s} ; \nu(\mathrm{CN}) 1502 \mathrm{~s}$. IR $\left(\mathrm{CH}_{2} \mathrm{Cl}_{2}, \mathrm{~cm}^{-1}\right): \nu(\mathrm{CO}) 2012 \mathrm{~s}$, $1920 \mathrm{~s} ; \nu(\mathrm{NO}) 1610 \mathrm{~s} ; \nu(\mathrm{CN}) 1486 \mathrm{~s}$.

\subsection{Synthesis of $[\mathrm{PPN}]\left[\mathrm{Mo}\left(\mathrm{Et}_{2} \mathrm{NCS}_{2}\right)(\mathrm{CO})_{2}(\mathrm{NO})(\mathrm{Cl})\right]$ (5)}

This yellow compound was prepared in a yield of $95 \%$ by a procedure similar to that used for 1 , using $\mathrm{PPN}^{+} \mathrm{Cl}^{-}$. Anal. Calc. for $\mathrm{C}_{43} \mathrm{H}_{40} \mathrm{ClMoN}_{3} \mathrm{O}_{3} \mathrm{P}_{2} \mathrm{~S}_{2}$ : C, 57.11; H, 4.46; N, 4.65. Found: C, 57.07; H, 4.50; N, 4.72\%. ${ }^{1} \mathrm{H}$ NMR $\left(23{ }^{\circ} \mathrm{C}\right.$, acetone-d $\left.{ }_{6}, 200 \mathrm{MHz}\right): \delta$, phenyl protons $7.70(\mathrm{~m}, 30 \mathrm{H}) ; \mathrm{Et}_{2} \mathrm{NCS}_{2}{ }^{-} 1.24(\mathrm{t}, 6 \mathrm{H}$, $\left.{ }^{3} J(\mathrm{H}, \mathrm{H})=7.2\right), 3.85(\mathrm{q}, 2 \mathrm{H}), 3.81(\mathrm{q}, 2 \mathrm{H}) . \mathrm{IR}(\mathrm{KBr}$, $\left.\mathrm{cm}^{-1}\right): \nu(\mathrm{CO}) 2000 \mathrm{~s}, 1912 \mathrm{~s} ; \nu(\mathrm{NO}) 1608 \mathrm{~s} ; \nu(\mathrm{CN}) 1502 \mathrm{~s}$. IR $\left(\mathrm{CH}_{2} \mathrm{Cl}_{2}, \mathrm{~cm}^{-1}\right): \nu(\mathrm{CO}) 2016 \mathrm{~s}, 1922 \mathrm{~s} ; \nu(\mathrm{NO}) 1604 \mathrm{~s}$; $\nu$ (CN) $1488 \mathrm{~s}$.

\subsection{Synthesis of $\left[\mathrm{Mo}\left(\mathrm{Et}_{2} \mathrm{NCS}_{2}\right)(\mathrm{CO})_{2}(\mathrm{NO})(\mathrm{py})\right]$ (6)}

To a $50 \mathrm{ml}$ round-bottom flask containing a magnetic bar, $0.16 \mathrm{~g}$ of $\left[\mathrm{Mo}\left(\mathrm{Et}_{2} \mathrm{NCS}_{2}\right)(\mathrm{CO})_{2}(\mathrm{NO})\right]_{2}(0.24 \mathrm{mmol})$ and $15 \mathrm{ml}$ of $\mathrm{CH}_{2} \mathrm{Cl}_{2}, 0.5 \mathrm{ml}$ of py was added and the resulting solution stirred for $15 \mathrm{~min}$ at room temperature. The solvents were then removed under vacuum. Recrystallization from $\mathrm{CH}_{2} \mathrm{Cl}_{2}$ /hexane gave an orange-red product $(0.17 \mathrm{~g}, 87 \%)$. Anal. Calc. for $\mathrm{C}_{12} \mathrm{H}_{16} \mathrm{MoN}_{3} \mathrm{O}_{3} \mathrm{~S}_{2}$ : C, 35.21; H, 3.69; N, 10.27. Found: C, 35.07; H, 3.73; $\mathrm{N}, 10.33 \% .{ }^{1} \mathrm{H}$ NMR $\left(23{ }^{\circ} \mathrm{C}\right.$, acetone- $\left.\mathrm{d}_{6}, 200 \mathrm{MHz}\right)$ : $\delta$, py $7.45(\mathrm{~m}, 2 \mathrm{H}), 8.03(\mathrm{~m}, 2 \mathrm{H}), 8.78(\mathrm{~m}, 1 \mathrm{H}) ; \mathrm{Et}_{2} \mathrm{NCS}_{2}{ }^{-}$ $1.23(\mathrm{~m}, 6 \mathrm{H}), 3.86(\mathrm{~m}, 2 \mathrm{H})$. IR $\left(\mathrm{KBr}, \mathrm{cm}^{-1}\right): \nu(\mathrm{CO})$ $2012 \mathrm{~s}, 1916 \mathrm{~s} ; \nu(\mathrm{NO}) 1644 \mathrm{~s}, 1602 \mathrm{~m} ; \nu(\mathrm{CN}) 1504 \mathrm{~s}$. IR $\left(\mathrm{CH}_{2} \mathrm{Cl}_{2}, \mathrm{~cm}^{-1}\right): \nu(\mathrm{CO}) 2020 \mathrm{~s}, 1924 \mathrm{~s} ; \nu(\mathrm{NO}) 1646 \mathrm{~s}$, $1602 \mathrm{~m} ; \nu(\mathrm{CN}) 1498 \mathrm{~s}$.

\subsection{Synthesis of $\left[\mathrm{Mo}\left(\mathrm{Et}_{2} \mathrm{NCS_{2 }}\right)(\mathrm{CO})(\mathrm{NO})(\mathrm{dppe})\right]$}

To a $50 \mathrm{ml}$ round-bottom flask containing a magnetic bar, $0.16 \mathrm{~g}$ of $\left[\mathrm{Mo}\left(\mathrm{Et}_{2} \mathrm{NCS}_{2}\right)(\mathrm{CO})_{2}(\mathrm{NO})\right]_{2}(0.24 \mathrm{mmol})$ and $0.96 \mathrm{~g}$ of dppe $(0.24 \mathrm{mmol})$ were added to $15 \mathrm{ml}$ of $\mathrm{CH}_{2} \mathrm{Cl}_{2}$. The resulting solution was stirred for 30 min at room temperature. The solvent was then removed under vacuum. Recrystallization from $\mathrm{CH}_{2} \mathrm{Cl}_{2} / \mathrm{Et}_{2} \mathrm{O}$ gave 
a red product $(0.28 \mathrm{~g}, 83 \%)$. Anal. Calc. for $\mathrm{C}_{32} \mathrm{H}_{34} \mathrm{MoN}_{2} \mathrm{O}_{2} \mathrm{P}_{2} \mathrm{~S}_{2}: \mathrm{C}, 54.86 ; \mathrm{H}, 4.89 ; \mathrm{N}, 4.00$. Found: $\mathrm{C}, 54.85 ; \mathrm{H}, 4.91 ; \mathrm{N}, 3.99 \% .{ }^{1} \mathrm{H}\left\{{ }^{31} \mathrm{P}\right\} \mathrm{NMR}\left(27{ }^{\circ} \mathrm{C}\right.$, acetone- $\left.\mathrm{d}_{6}, 400 \mathrm{MHz}\right): \delta$, dppe $7.55(\mathrm{~m}, 40 \mathrm{H}), 2.66(\mathrm{~m}$, $8 \mathrm{H}) ; \mathrm{Et}_{2} \mathrm{NCS}_{2}-1.08\left(\mathrm{t}, 3 \mathrm{H},{ }^{3} J(\mathrm{H}, \mathrm{H})=7.2\right), 1.00(\mathrm{t}, 3 \mathrm{H}$, $\left.{ }^{3} J(\mathrm{H}, \mathrm{H})=7.2\right), 0.88\left(\mathrm{t}, 3 \mathrm{H},{ }^{3} J(\mathrm{H}, \mathrm{H})=7.2\right), 0.75(\mathrm{t}, 3 \mathrm{H}$, $\left.{ }^{3} J(\mathrm{H}, \mathrm{H})=7.2\right), 3.55(\mathrm{~m}, 4 \mathrm{H}), 3.23(\mathrm{~m}, 4 \mathrm{H}) .{ }^{31} \mathrm{P}\left\{{ }^{1} \mathrm{H}\right\}$ NMR $\left(27{ }^{\circ} \mathrm{C}\right.$, acetone-d $\left.{ }_{6}, 162 \mathrm{MHz}\right): \delta$, dppe 67.76, 64.22, 41.10, 23.07. IR (KBr, $\left.\mathrm{cm}^{-1}\right): \nu(\mathrm{CO}) 1898 \mathrm{~s}, 1872 \mathrm{~s}$; $\nu$ (NO) $1602 \mathrm{~s}, 1580 \mathrm{~m} ; \nu(\mathrm{CN}) 1488 \mathrm{~s}$. IR $\left(\mathrm{CH}_{2} \mathrm{Cl}_{2}, \mathrm{~cm}^{-1}\right)$ : $\nu$ (CO) 1912 (s, br); $\nu$ (NO) 1600 (s, br); $\nu$ (CN) 1488 s.

\subsection{Synthesis of $\left[\mathrm{Mo}\left(\mathrm{Et}_{2} \mathrm{NCS}_{2}\right)(\mathrm{CO})(\mathrm{NO})\left(\mathrm{PPh}_{3}\right)_{2}\right]$}

This red compound was prepared in a yield of $85 \%$ by a procedure similar to that used for 1 , using 4 equiv. of $\mathrm{PPh}_{3}$. Anal. Calc. for $\mathrm{C}_{42} \mathrm{H}_{40} \mathrm{MoN}_{2} \mathrm{O}_{2} \mathrm{P}_{2} \mathrm{~S}_{2}: \mathrm{C}, 61.01$; H, 4.88; N, 3.39. Found: C, 60.87; H, 4.86; N, 3.34\%. ${ }^{1} \mathrm{H}$ NMR $\left(27{ }^{\circ} \mathrm{C}, \mathrm{CDCl}_{3}, 200 \mathrm{MHz}\right): \delta, \mathrm{PPh}_{3} 7.45(\mathrm{~m}$, $30 \mathrm{H}) ; \mathrm{Et}_{2} \mathrm{NCS}_{2}{ }^{-} 0.62\left(\mathrm{t}, 3 \mathrm{H},{ }^{3} \mathrm{~J}(\mathrm{H}, \mathrm{H})=7.1\right), 0.49(\mathrm{t}$, $\left.3 \mathrm{H},{ }^{3} J(\mathrm{H}, \mathrm{H})=7.1\right), 2.97(\mathrm{q}, 2 \mathrm{H}), 2.76(\mathrm{q}, 2 \mathrm{H}) .{ }^{31} \mathrm{P}\left\{{ }^{1} \mathrm{H}\right\}$ NMR $\left(27{ }^{\circ} \mathrm{C}, \mathrm{CDCl}_{3}, 162 \mathrm{MHz}\right): \delta, \mathrm{PPh}_{3}$ 43.08. IR $\left(\mathrm{KBr}, \mathrm{cm}^{-1}\right): \nu(\mathrm{CO}) 1888 \mathrm{~s} ; \nu(\mathrm{NO}) 1604 \mathrm{~s} ; \nu(\mathrm{CN}) 1484 \mathrm{~s}$. IR $\left(\mathrm{CH}_{2} \mathrm{Cl}_{2}, \mathrm{~cm}^{-1}\right): \nu(\mathrm{CO}) 1904 \mathrm{~s} ; \nu(\mathrm{NO}) 1606 \mathrm{~s} ; \nu(\mathrm{CN})$ $1486 s$.

\subsection{Synthesis of $\left[\mathrm{Mo}\left(\mathrm{Et}_{2} \mathrm{NCS}_{2}\right)(\mathrm{CO})(\mathrm{NO})\left(\mathrm{PMe}_{3}\right)_{2}\right]$}

(9)

This red compound was prepared in a yield of $75 \%$ by a procedure similar to that used for 1 , using 1.5 $\mathrm{ml}$ of $\mathrm{PMe}_{3}(1.0 \mathrm{M}$ solution in toluene). Anal. Calc. for $\mathrm{C}_{12} \mathrm{H}_{28} \mathrm{MoN}_{2} \mathrm{O}_{2} \mathrm{P}_{2} \mathrm{~S}_{2}: \mathrm{C}, 31.72 ; \mathrm{H}, 6.21 ; \mathrm{N}, 6.17$. Found: C, 31.70; H, 6.19; N, 6.25\%. ${ }^{1} \mathrm{H}$ NMR $\left(27{ }^{\circ} \mathrm{C}\right.$, acetone- $\left.\mathrm{d}_{6}, 200 \mathrm{MHz}\right): \delta, \mathrm{PMe}_{3} 1.45(\mathrm{t}, \quad 18 \mathrm{H}$, $\left.{ }^{2} J(\mathrm{P}, \mathrm{H})={ }^{4} J(\mathrm{P}, \mathrm{H})=3.3\right) ; \quad \mathrm{Et}_{2} \mathrm{NCS}_{2}{ }^{-} \quad 1.25 \quad(\mathrm{t}, \quad 3 \mathrm{H}$, $\left.{ }^{3} J(\mathrm{H}, \mathrm{H})=7.1\right), 1.78\left(\mathrm{t}, 3 \mathrm{H},{ }^{3} J(\mathrm{H}, \mathrm{H})=7.1\right), 3.89(\mathrm{q}, 2 \mathrm{H})$, $3.76(\mathrm{q}, 2 \mathrm{H}) .{ }^{31} \mathrm{P}\left\{{ }^{1} \mathrm{H}\right\} \mathrm{NMR}\left(27^{\circ} \mathrm{C}, \mathrm{CDCl}_{3}, 162 \mathrm{MHz}\right)$ : $\delta, \mathrm{PMe}_{3}-6.62$. IR $\left(\mathrm{KBr}, \mathrm{cm}^{-1}\right): \nu(\mathrm{CO}) 1874 \mathrm{~s} ; \nu(\mathrm{NO})$ 1596s; $\nu(\mathrm{CN}) 1496 \mathrm{~s}$. IR $\left(\mathrm{CH}_{2} \mathrm{Cl}_{2}, \mathrm{~cm}^{-1}\right): \nu(\mathrm{CO}) 1874 \mathrm{~s}$; $\nu(\mathrm{NO}) 1600 \mathrm{~s} ; \nu(\mathrm{CN}) 1488 \mathrm{~s}$.

\subsection{Synthesis of \\ $\left[\mathrm{Mo}\left(\mathrm{Et}_{2} \mathrm{NCS}_{2}\right)(\mathrm{CO})(\mathrm{NO})\left\{\mathrm{P}(\mathrm{OMe})_{3}\right\}_{2}\right]$}

This red compound was prepared in a yield of $75 \%$ by a procedure similar to that used for 1 , using 0.5 $\mathrm{ml}$ of $\mathrm{P}(\mathrm{OMe})_{3}$. Anal. Calc. for $\mathrm{C}_{12} \mathrm{H}_{28} \mathrm{MoN}_{2} \mathrm{O}_{8} \mathrm{P}_{2} \mathrm{~S}_{2}: \mathrm{C}$, 26.19; H, 5.13; N, 5.09. Found: C, 26.13; H, 5.13; N, 5.18\%. ${ }^{1} \mathrm{H}$ NMR $\left(27^{\circ} \mathrm{C}, \mathrm{CDCl}_{3}, 200 \mathrm{MHz}\right): \delta, \mathrm{P}(\mathrm{OMe})_{3}$ $3.72\left(\mathrm{t}, 18 \mathrm{H},{ }^{3} \mathrm{~J}(\mathrm{P}, \mathrm{H})={ }^{5} \mathrm{~J}(\mathrm{P}, \mathrm{H})=2.7\right) ; \mathrm{Et}_{2} \mathrm{NCS}_{2}{ }^{-}, 1.17$ $\left(\mathrm{t}, 3 \mathrm{H},{ }^{3} \mathrm{~J}(\mathrm{H}, \mathrm{H})=7.1\right), 1.25\left(\mathrm{t}, 3 \mathrm{H},{ }^{3} \mathrm{~J}(\mathrm{H}, \mathrm{H})=7.1\right), 3.81$ (q, 2H), 3.67 (q, 2H). ${ }^{31} \mathrm{P}\left\{{ }^{1} \mathrm{H}\right\}$ NMR $\left(27{ }^{\circ} \mathrm{C}, \mathrm{CDCl}_{3}\right.$, $162 \mathrm{MHz}): \delta, \mathrm{P}(\mathrm{OMe})_{3}$ 159.30. IR $\left(\mathrm{KBr}, \mathrm{cm}^{-1}\right): \nu(\mathrm{CO})$
$1924 \mathrm{~s} ; \nu(\mathrm{NO}) 1626 \mathrm{~s} ; \nu(\mathrm{CN}) 1502 \mathrm{~s}$. IR $\left(\mathrm{CH}_{2} \mathrm{Cl}_{2}, \mathrm{~cm}^{-1}\right)$ : $\nu(\mathrm{CO}) 1922 \mathrm{~s} ; \nu(\mathrm{NO}) 1626 \mathrm{~s} ; \nu(\mathrm{CN}) 1494 \mathrm{~s}$.

\subsection{X-ray diffraction study}

Single crystals were grown by the liquid diffusion method from $\mathrm{CH}_{2} \mathrm{Cl}_{2} /$ hexane at room temperature. The intensity data for suitable crystals of the complexes were collected at room temperature on a CAD-4 diffractometer using monochromated $\mathrm{Mo} \mathrm{K} \alpha$ radiation $(\lambda=0.710930 \AA)$. The unit-cell constants were derived from a least-squares refinement of 24 setting reflections. The $\theta-2 \theta$ scan technique and a variable scan speed were used to obtain the integrated intensities. Three reference reflections were monitored throughout the measurement and the variation of the intensities was a total decay of less than $2 \%$ in any complex. Absorption corrections were applied for each structure according to experimental $\psi$ rotation curves. The structural analyses were carried out on Microvax III using NRCVAX programs [4]. Atomic scattering factors were taken from the literature [5].

The metal atom position in any of the seven structures reported here was first determined by the heavy atom method. The remaining non-hydrogen atoms were subsequently located from the Fourier difference maps and all atoms except the disordered atom were then refined anisotropically $\left(\mathrm{C}(12)\right.$ and $\mathrm{C}\left(12^{\prime}\right)$ each with occupancy $=0.50$ in structure 10 were found disordered). We have checked the transformed cells of both 3 and 8 crystals, by transformation matrix $1001020-10$ and 100102010 , respectively, and found that they are not satisfactory. First, the $\gamma$ angles deviate from $90.0^{\circ}$. Second, the intensities of $h k l$ do not equal to those of $h \bar{k} l$. The ambiguities of locating NO and CO positions, if there are any, were solved according to the chemical and crystallographical meaningfulness of thermal displacement coefficients. A more restricted $\mathrm{N}$ atomic peak with a lower thermal parameter, $U$, than the neighboring $O$ atomic peak [6] confirmed the successful assignment of the NO positions. Accordingly, only one nitrosyl position was found for any of the seven structures described here. The positions of all hydrogen atoms were calculated and refined isotropically to give residual $R$ and $R_{\mathrm{w}}$ values shown in Table 1 with other crystallographic information.

In the asymmetric unit of the crystal used for $\mathbf{8}$, there is a half molecule of $\mathrm{CH}_{2} \mathrm{Cl}_{2}$. The ORTEP plots for anions of 1 (Fig. 1), 3 (Fig. 2) and 4 (Fig. 3), and neutral molecules of 6 (Fig. 4), 7 (Fig. 5), 8 (Fig. 6) and 10 (Fig. 7) are shown with $50 \%$ probability thermal ellipsoids. The numbering schemes in the figures correspond to the atomic positions (Table 2). Some selected bond lengths and angles are collected in Table 3. See also Section 4. 


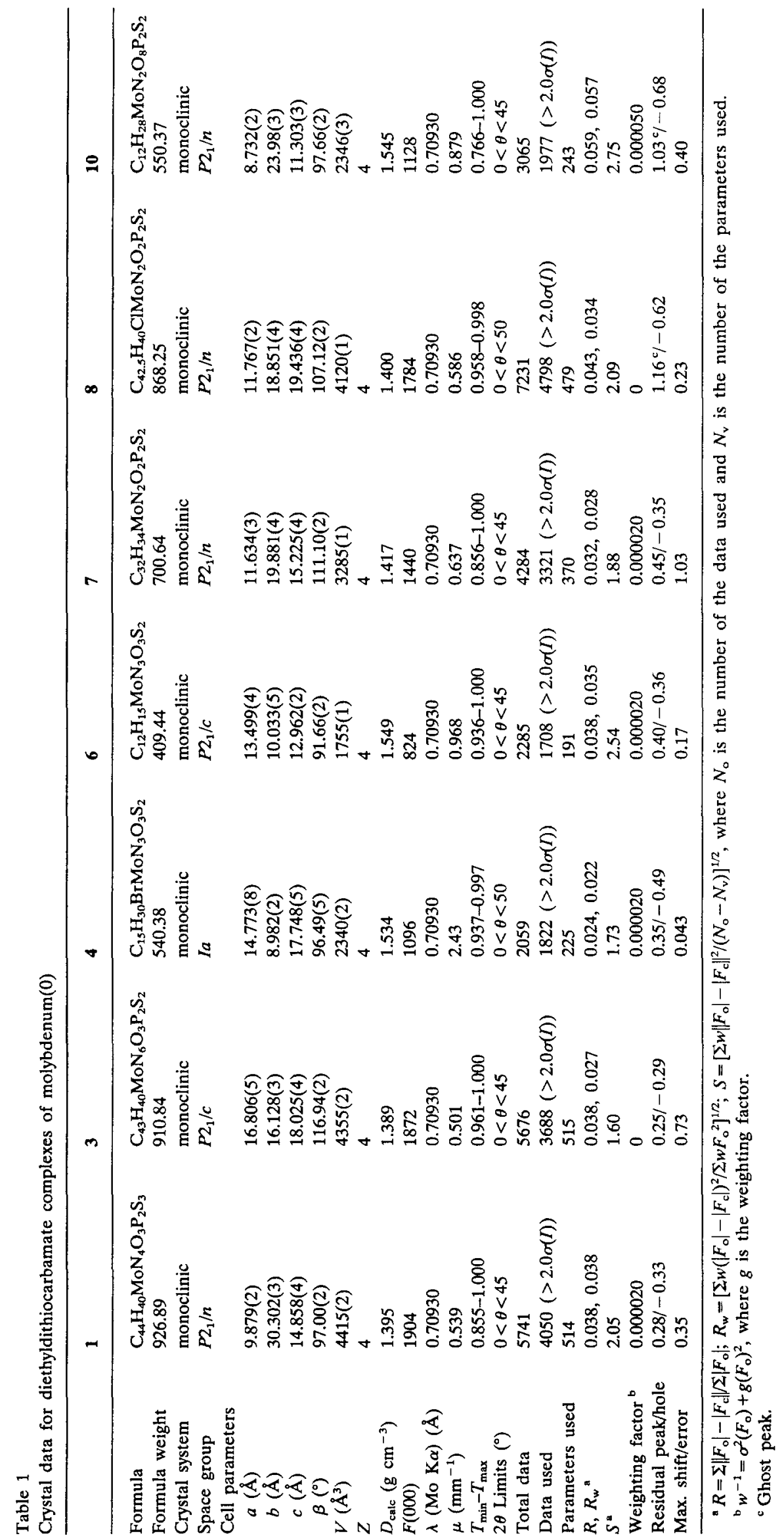


Table 2

Fractional atomic coordinates and $B_{\varepsilon q}$ values

\begin{tabular}{lllll}
\hline Atom $x$ & $y$ & $z$ & $B_{\mathrm{eq}}{ }^{\mathrm{a}}$ \\
\hline
\end{tabular}

(a) $[\mathrm{PPN}]\left[\mathrm{Mo}\left(\mathrm{Et}_{2} \mathrm{NCS}_{2}\right)(\mathrm{CO})_{2}(\mathrm{NO})(\mathrm{NCS})\right]$ (1)

\begin{tabular}{|c|c|c|c|c|c|c|c|c|c|}
\hline \multicolumn{5}{|l|}{ ractic } & & & & & \\
\hline & & & & & Ato & $x$ & $y$ & $z$ & $B_{\text {eq }}{ }^{2}$ \\
\hline & & $y$ & 2 & $D_{\text {eq }}$ & \multirow{2}{*}{$\begin{array}{l}\text { N2 } \\
\text { N3 }\end{array}$} & \multirow{2}{*}{$\begin{array}{l}0.4124(4) \\
0.4720(4)\end{array}$} & \multirow{2}{*}{$\begin{array}{l}0.2755(3) \\
0.3003(4)\end{array}$} & \multirow{2}{*}{$\begin{array}{l}0.3222(4) \\
0.3811(4)\end{array}$} & \multirow{2}{*}{$\begin{array}{l}4.7(4) \\
7.6(4)\end{array}$} \\
\hline \multicolumn{5}{|c|}{ (a) $[\mathrm{PPN}]\left[\mathrm{Mo}\left(\mathrm{Et}_{2} \mathrm{NCS}_{2}\right)(\mathrm{CO})_{2}(\mathrm{NO})(\mathrm{NCS})\right]$ (1) } & & & & & \\
\hline Mo & $0.13725(5)$ & $0.11904(2)$ & $0.13528(3)$ & $3.52(3)$ & N4 & $0.1237(3)$ & $0.1421(3)$ & $0.2147(3)$ & $3.6(3)$ \\
\hline S1 & $0.2655(2)$ & $0.19142(5)$ & $0.1642(1)$ & $3.96(8)$ & O4 & $0.0556(3)$ & $0.1054(3)$ & $0.1958(3)$ & $5.1(3)$ \\
\hline S2 & $0.3820(2)$ & $0.10506(5)$ & $0.2030(1)$ & $4.14(8)$ & $\mathrm{Cl}$ & $0.2443(4)$ & $0.1140(4)$ & $0.1641(4)$ & $4.1(4)$ \\
\hline S3 & $0.0230(2)$ & $0.11011(6)$ & $0.4495(1)$ & $4.77(9)$ & O1 & $0.2502(3)$ & $0.0664(3)$ & $0.1200(3)$ & $6.1(3)$ \\
\hline $\mathrm{C} 1$ & $0.0592(5)$ & $0.1163(2)$ & $0.3470(4)$ & $3.2(3)$ & $\mathrm{C} 2$ & $0.1717(4)$ & $0.2817(4)$ & $0.1556(4)$ & $4.3(4)$ \\
\hline N1 & $0.0865(5)$ & $0.1204(2)$ & $0.2740(3)$ & $4.0(3)$ & $\mathrm{O} 2$ & $0.1405(3)$ & $0.3347(3)$ & $0.1092(3)$ & $6.6(3)$ \\
\hline $\mathrm{C} 2$ & $0.4083(6)$ & $0.1609(2)$ & $0.2017(4)$ & $3.5(3)$ & $\mathrm{C} 3$ & $0.2982(3)$ & $0.1987(4)$ & $0.4256(3)$ & $3.3(3)$ \\
\hline N2 & $0.5287(5)$ & $0.1790(2)$ & $0.2287(3)$ & $3.7(2)$ & N5 & $0.3328(3)$ & $0.2019(3)$ & $0.5084(3)$ & $3.7(3)$ \\
\hline $\mathrm{C} 3$ & $0.6453(6)$ & $0.1523(2)$ & $0.2675(5)$ & $5.1(4)$ & $\mathrm{C} 4$ & $0.3766(4)$ & $0.1292(4)$ & $0.5605(4)$ & $5.0(4)$ \\
\hline $\mathrm{C} 4$ & $0.6449(7)$ & $0.1445(3)$ & $0.3674(5)$ & $6.7(4)$ & $\mathrm{C} 5$ & $0.3103(5)$ & $0.0730(5)$ & $0.5695(4)$ & $6.9(5)$ \\
\hline $\mathrm{C} 5$ & $0.5522(6)$ & $0.2264(2)$ & $0.2234(5)$ & $4.8(4)$ & $\mathrm{C} 6$ & $0.3288(4)$ & $0.2768(4)$ & $0.5521(4)$ & $4.5(4)$ \\
\hline $\mathrm{C} 6$ & $0.6246(7)$ & $0.2388(2)$ & $0.1433(5)$ & $6.5(4)$ & $\mathrm{C} 7$ & $0.4109(5)$ & $0.3288(4)$ & $0.5766(4)$ & $6.5(5)$ \\
\hline N3 & $0.1625(4)$ & $0.1130(2)$ & $0.0192(3)$ & $3.9(3)$ & P1 & $0.2801(1)$ & $0.6990(1)$ & $0.18466(9)$ & $2.87(8)$ \\
\hline $\mathrm{O} 1$ & $0.1706(4)$ & $0.1072(2)$ & $-0.0599(3)$ & $5.9(3)$ & P2 & $0.1875(1)$ & $0.78094(9)$ & $0.02154(9)$ & $2.83(8)$ \\
\hline $\mathrm{C} 7$ & $0.0689(7)$ & $0.0569(2)$ & $0.1256(4)$ & $4.3(3)$ & N6 & $0.2670(3)$ & $0.7447(3)$ & $0.1030(3)$ & $3.2(3)$ \\
\hline $\mathrm{O} 2$ & $0.0319(5)$ & $0.0214(2)$ & $0.1132(3)$ & $6.6(3)$ & $\mathrm{C} 11 \mathrm{~A}$ & $0.3192(4)$ & $0.7695(4)$ & $0.2712(4)$ & $2.9(3)$ \\
\hline $\mathrm{C} 8$ & $-0.0506(7)$ & $0.1413(2)$ & $0.0972(4)$ & $4.4(3)$ & $\mathrm{C} 12 \mathrm{~A}$ & $0.3200(4)$ & $0.8539(4)$ & $0.2583(4)$ & $4.1(4)$ \\
\hline $\mathrm{O} 3$ & $-0.1586(5)$ & $0.1535(2)$ & $0.0735(4)$ & $7.1(3)$ & $\mathrm{C} 13 \mathrm{~A}$ & $0.3522(5)$ & $0.9077(4)$ & $0.3241(5)$ & $5.6(5)$ \\
\hline $\mathrm{P} 1$ & $0.9722(1)$ & $0.43299(5)$ & $0.2227(1)$ & $2.66(6)$ & $\mathrm{C} 14 \mathrm{~A}$ & $0.3850(5)$ & $0.8785(5)$ & $0.4031(5)$ & $5.8(5)$ \\
\hline $\mathbf{P} 2$ & $1.0119(1)$ & $0.33664(5)$ & $0.1964(1)$ & $2.68(6)$ & C15A & $0.3854(4)$ & $0.7956(5)$ & $0.4187(4)$ & $5.3(5)$ \\
\hline N4 & $0.9421(4)$ & $0.3834(1)$ & $0.1951(3)$ & $3.0(2)$ & C16A & $0.3521(4)$ & $0.7403(4)$ & $0.3520(4)$ & $4.5(4)$ \\
\hline $\mathrm{C} 11 \mathrm{~A}$ & $0.8098(5)$ & $0.4609(2)$ & $0.2128(3)$ & $2.6(3)$ & C11B & $0.1809(4)$ & $0.6484(4)$ & $0.1768(3)$ & $3.1(3)$ \\
\hline $\mathrm{C} 12 \mathrm{~A}$ & $0.6930(6)$ & $0.4387(2)$ & $0.1791(4)$ & $3.6(3)$ & $\mathrm{C} 12 \mathrm{~B}$ & $0.1301(4)$ & $0.6853(4)$ & $0.2106(3)$ & $4.2(4)$ \\
\hline $\mathrm{C} 13 \mathrm{~A}$ & $0.5695(6)$ & $0.4611(2)$ & $0.1659(4)$ & $4.4(3)$ & C13B & $0.0489(5)$ & $0.6500(5)$ & $0.1952(4)$ & $5.9(5)$ \\
\hline $\mathrm{C} 14 \mathrm{~A}$ & $0.5634(6)$ & $0.5050(2)$ & $0.1871(4)$ & $4.3(3)$ & C14B & $0.0199(5)$ & $0.5796(5)$ & $0.1475(5)$ & $6.8(6)$ \\
\hline $\mathrm{C} 15 \mathrm{~A}$ & $0.6792(6)$ & $0.5268(2)$ & $0.2222(4)$ & $4.2(3)$ & C15B & $0.0707(5)$ & $0.5422(4)$ & $0.1155(5)$ & $6.4(5)$ \\
\hline C16A & $0.8026(6)$ & $0.5054(2)$ & $0.2344(4)$ & $3.6(3)$ & $\mathrm{C} 16 \mathrm{~B}$ & $0.1511(4)$ & $0.5771(4)$ & $0.1302(4)$ & $4.9(5)$ \\
\hline $\mathrm{C} 11 \mathrm{~B}$ & $1.0523(6)$ & $0.4411(2)$ & $0.3377(4)$ & $3.1(3)$ & $\mathrm{C} 11 \mathrm{C}$ & $0.3651(4)$ & $0.6209(3)$ & $0,2100(3)$ & $2.8(3)$ \\
\hline $\mathrm{C} 12 \mathrm{~B}$ & $0.9731(7)$ & $0.4387(2)$ & $0.4071(4)$ & $4.8(3)$ & $\mathrm{C} 12 \mathrm{C}$ & $0.3698(4)$ & $0.5534(4)$ & $0.2591(4)$ & $3.9(4)$ \\
\hline C13B & $1.0344(9)$ & $0.4407(3)$ & $0.4959(5)$ & $6.6(5)$ & $\mathrm{C} 13 \mathrm{C}$ & $0.4387(5)$ & $0.4963(4)$ & $0.2813(4)$ & $5.0(5)$ \\
\hline $\mathrm{C} 14 \mathrm{~B}$ & $1.173(1)$ & $0.4442(3)$ & $0.5136(5)$ & $7.2(5)$ & $\mathrm{C} 14 \mathrm{C}$ & $0.5015(5)$ & $0.5085(5)$ & $0.2544(5)$ & $5.4(4)$ \\
\hline C15B & $1.2530(7)$ & $0.4467(3)$ & $0.4450(5)$ & $6.0(4)$ & $\mathrm{C} 15 \mathrm{C}$ & $0.4981(4)$ & $0.5751(5)$ & $0.2071(4)$ & $4.9(5)$ \\
\hline $\mathrm{C} 16 \mathrm{~B}$ & $1.1926(6)$ & $0.4445(2)$ & $0.3561(4)$ & $4.4(3)$ & $\mathrm{C} 16 \mathrm{C}$ & $0.4302(4)$ & $0.6318(4)$ & $0.1844(4)$ & $3.8(4)$ \\
\hline $\mathrm{C} 11 \mathrm{C}$ & $1.0762(5)$ & $0.4604(2)$ & $0.1491(3)$ & $2.7(3)$ & $\mathrm{C} 21 \mathrm{~A}$ & $0.0961(3)$ & $0.8220(4)$ & $0.0359(3)$ & $2.8(3)$ \\
\hline $\mathrm{C} 12 \mathrm{C}$ & $1.1246(6)$ & $0.5029(2)$ & $0.1691(4)$ & $3.6(3)$ & $\mathrm{C} 22 \mathrm{~A}$ & $0.0989(4)$ & $0.9017(4)$ & $0.0633(4)$ & $3.9(4)$ \\
\hline $\mathrm{C} 13 \mathrm{C}$ & $1.1989(6)$ & $0.5245(2)$ & $0.1100(5)$ & $4.4(3)$ & $\mathrm{C} 23 \mathrm{~A}$ & $0.0332(5)$ & $0.9316(4)$ & $0.0812(4)$ & $5.3(5)$ \\
\hline $\mathrm{C} 14 \mathrm{C}$ & $1.2260(6)$ & $0.5044(3)$ & $0.0322(4)$ & $4.9(4)$ & $\mathrm{C} 24 \mathrm{~A}$ & $-0.0374(5)$ & $0.8827(5)$ & $0.0701(4)$ & $5.5(5)$ \\
\hline $\mathrm{C} 15 \mathrm{C}$ & $1.1787(7)$ & $0.4625(3)$ & $0.0112(4)$ & $5.4(4)$ & $\mathrm{C} 25 \mathrm{~A}$ & $-0.0411(4)$ & $0.8036(5)$ & $0.0440(4)$ & $5.2(5)$ \\
\hline $\mathrm{C} 16 \mathrm{C}$ & $1.1021(6)$ & $0.4412(2)$ & $0.0691(4)$ & $3.9(3)$ & $\mathrm{C} 26 \mathrm{~A}$ & $0.0252(4)$ & $0.7716(4)$ & $0.0263(3)$ & $3.8(4)$ \\
\hline $\mathrm{C} 21 \mathrm{~A}$ & $1.1953(5)$ & $0.3374(2)$ & $0.2163(4)$ & $2.9(3)$ & $\mathrm{C} 21 \mathrm{~B}$ & $0.1437(4)$ & $0.7050(3)$ & $-0.0604(3)$ & $2.9(3)$ \\
\hline $\mathrm{C} 22 \mathrm{~A}$ & $1.2576(6)$ & $0.3388(2)$ & $0.3060(4)$ & $3.7(3)$ & $\mathrm{C} 22 \mathrm{~B}$ & $0.1955(4)$ & $0.6364(4)$ & $-0.0570(4)$ & $3.8(4)$ \\
\hline $\mathrm{C} 23 \mathrm{~A}$ & $1.3978(6)$ & $0.3441(2)$ & $0.3230(4)$ & $4.5(3)$ & $\mathrm{C} 23 \mathrm{~B}$ & $0.1651(5)$ & $0.5814(4)$ & $-0.1229(5)$ & $5.2(5)$ \\
\hline $\mathrm{C} 24 \mathrm{~A}$ & $1.4751(6)$ & $0.3468(2)$ & $0.2539(5)$ & $5.1(4)$ & $\mathrm{C} 24 \mathrm{~B}$ & $0.0845(5)$ & $0.5935(4)$ & $-0.1911(4)$ & $5.1(5)$ \\
\hline $\mathrm{C} 25 \mathrm{~A}$ & $1.4142(6)$ & $0.3446(2)$ & $0.1652(4)$ & $5.1(4)$ & $\mathrm{C} 25 \mathrm{~B}$ & $0.0325(4)$ & $0.6597(4)$ & $-0.1947(4)$ & $4.9(4)$ \\
\hline $\mathrm{C} 26 \mathrm{~A}$ & $1.2750(6)$ & $0.3401(2)$ & $0.1472(4)$ & $3.7(3)$ & $\mathrm{C} 26 \mathrm{~B}$ & $0.0614(4)$ & $0.7158(4)$ & $-0.1302(4)$ & $4.0(4)$ \\
\hline $\mathrm{C} 21 \mathrm{~B}$ & $0.9634(5)$ & $0.3104(2)$ & $0.0895(3)$ & $2.8(3)$ & $\mathrm{C} 21 \mathrm{C}$ & $0.2314(4)$ & $0.8644(3)$ & $-0.0147(3)$ & $2.9(3)$ \\
\hline $\mathrm{C} 22 \mathrm{~B}$ & $1.0251(6)$ & $0.2713(2)$ & $0.0658(4)$ & $4.1(3)$ & $\mathrm{C} 22 \mathrm{C}$ & $0.1757(4)$ & $0.9119(4)$ & $-0.0826(4)$ & $3.9(4)$ \\
\hline $\mathrm{C} 23 \mathrm{~B}$ & $0.9805(7)$ & $0.2507(2)$ & $-0.0149(4)$ & $4.6(3)$ & $\mathrm{C} 23 \mathrm{C}$ & $0.2099(4)$ & $0.9751(4)$ & $-0.1104(4)$ & $4.3(4)$ \\
\hline $\mathrm{C} 24 \mathrm{~B}$ & $0.8767(7)$ & $0.2688(2)$ & $-0.0735(4)$ & $4.7(3)$ & $\mathrm{C} 24 \mathrm{C}$ & $0.3001(5)$ & $0.9917(4)$ & $-0.0711(4)$ & $4.3(4)$ \\
\hline $\mathrm{C} 25 \mathrm{~B}$ & $0.8183(6)$ & $0.3076(2)$ & $-0.0530(4)$ & $4.9(4)$ & $\mathrm{C} 25 \mathrm{C}$ & $0.3554(4)$ & $0.9451(4)$ & $-0.0052(4)$ & $4.7(4)$ \\
\hline $\mathrm{C} 26 \mathrm{~B}$ & $0.8582(6)$ & $0.3286(2)$ & $0.0294(4)$ & $4.0(3)$ & $\mathrm{C} 26 \mathrm{C}$ & $0.3222(4)$ & $0.8809(4)$ & $0.0237(3)$ & $3.7(4)$ \\
\hline $\mathrm{C} 21 \mathrm{C}$ & $0.9544(5)$ & $0.3025(2)$ & $0.2845(4)$ & $2.8(3)$ & \multirow{2}{*}{\multicolumn{5}{|c|}{ (c) $\left[\mathrm{Et}_{4} \mathrm{~N}\right]\left[\mathrm{Mo}\left(\mathrm{Et}_{2} \mathrm{NCS}_{2}\right)(\mathrm{CO})_{2}(\mathrm{NO})(\mathrm{Br})\right]$ (4) }} \\
\hline $\mathrm{C} 22 \mathrm{C}$ & $0.9943(6)$ & $0.2590(2)$ & $0.2954(4)$ & $4.3(3)$ & & & & & $2.85(2)$ \\
\hline $\mathrm{C} 23 \mathrm{C}$ & $0.9518(7)$ & $0.2340(2)$ & $0.3638(5)$ & $5.2(4)$ & $\begin{array}{l}\mathrm{Mo} \\
\mathrm{Br}\end{array}$ & $\begin{array}{l}0.30830 \\
0.30139(7)\end{array}$ & $\begin{array}{l}0.9950 /(5) \\
0.70062(6)\end{array}$ & $0.13508(6)$ & $\begin{array}{l}2.85(2) \\
4.08(3)\end{array}$ \\
\hline $\mathrm{C} 24 \mathrm{C}$ & $0.8717(7)$ & $0.2522(2)$ & $0.4227(4)$ & $5.0(4)$ & $\begin{array}{l}\mathrm{Br} \\
\mathrm{S} 1\end{array}$ & $0.3999(1)$ & $1.0082(2)$ & $0.03845(9)$ & $\begin{array}{l}4.08(3) \\
3.61(7)\end{array}$ \\
\hline $\mathrm{C} 25 \mathrm{C}$ & $0.8332(7)$ & $0.2955(3)$ & $0.4124(5)$ & $5.7(4)$ & $\begin{array}{l}\text { S1 } \\
\text { S2 }\end{array}$ & $0.2028(1)$ & $1.0211(2)$ & $\begin{array}{l}0.03040(9) \\
0.0270(1)\end{array}$ & $\begin{array}{l}3.61(1) \\
4.04(8)\end{array}$ \\
\hline $\mathrm{C} 26 \mathrm{C}$ & $0.8732(6)$ & $0.3207(2)$ & $0.3433(4)$ & $4.1(3)$ & $\begin{array}{l}\text { S2 } \\
\mathrm{C}\end{array}$ & $\begin{array}{l}0.2028(1) \\
0.2984(4)\end{array}$ & $\begin{array}{l}1.0211(2) \\
1.0219(7)\end{array}$ & $-0.0190(3)$ & $\begin{array}{l}3.04(0) \\
3.6(3)\end{array}$ \\
\hline \multicolumn{5}{|c|}{ (b) $[\mathrm{PPN}]\left[\mathrm{Mo}\left(\mathrm{Et}_{2} \mathrm{NCS}_{2}\right)(\mathrm{CO})_{2}(\mathrm{NO})\left(\eta^{1}-\mathrm{N}_{3}\right)\right](3)$} & N1 & $0.2953(4)$ & $1.0329(6)$ & $-0.0938(3)$ & $4.7(3)$ \\
\hline Mo & $0.22821(4)$ & $0.19321(3)$ & $0.24184(3)$ & $3.30(3)$ & $\mathrm{C} 1$ & $0.3781(6)$ & $1.0342(9)$ & $-0.1330(4)$ & $6.6(4)$ \\
\hline S1 & $0.3126(1)$ & $0.1142(1)$ & $0.3759(1)$ & $4.0(1)$ & $\mathrm{C} 2$ & $0.4028(9)$ & $0.884(1)$ & $-0.1547(6)$ & $10.6(7)$ \\
\hline S2 & $0.2366(1)$ & $0.2781(1)$ & $0.3630(1)$ & $4.2(1)$ & C3 & $0.2079(6)$ & $1.047(1)$ & $-0.1436(5)$ & $6.0(4)$ \\
\hline N1 & $0.3542(3)$ & $0.2512(3)$ & $0.2611(3)$ & $4.5(3)$ & $\mathrm{C} 4$ & $0.1807(6)$ & $1.210(1)$ & $-0.1506(5)$ & $7.3(5)$ \\
\hline
\end{tabular}

Table 2 (continued) 
Table 2 (continued)

\begin{tabular}{|c|c|c|}
\hline Atom & $x$ & $y$ \\
\hline C5 & $0.4105(5)$ & $0.9627(7)$ \\
\hline 05 & $0.4681(4)$ & $0.9485(7)$ \\
\hline C6 & $0.2211(5)$ & $0.9548(7)$ \\
\hline O6 & $0.1753(4)$ & $0.9333(7)$ \\
\hline N7 & $0.3079(3)$ & $1.1890(6)$ \\
\hline 07 & $0.3055(3)$ & $1.3148(5)$ \\
\hline N3 & $0.5284(3)$ & $0.4698(5)$ \\
\hline $\mathrm{CB}$ & $0.4804(4)$ & $0.4024(7)$ \\
\hline $\mathrm{CP}$ & $0.5412(6)$ & $0.3324(9)$ \\
\hline $\mathrm{C} 10$ & $0.6004(4)$ & $0.5823(7)$ \\
\hline $\mathrm{C} 11$ & $0.5676(6)$ & $0.703(1)$ \\
\hline $\mathrm{C} 12$ & $0.4601(4)$ & $0.5495(7)$ \\
\hline $\mathrm{C} 13$ & $0.3840(6)$ & $0.451(1)$ \\
\hline $\mathrm{C} 14$ & $0.5757(4)$ & $0.3450(7)$ \\
\hline $\mathrm{C} 15$ & $0.6187(6)$ & $0.3859(9)$ \\
\hline \multicolumn{3}{|c|}{ (d) $\left[\mathrm{Mo}\left(\mathrm{Et}_{2} \mathrm{NCS}_{2}\right)(\mathrm{CO})_{2}(\mathrm{NO})(\mathrm{py})\right](6)$} \\
\hline Mo & $0.30211(4)$ & $0.18907(6)$ \\
\hline $\mathrm{S} 1$ & $0.1313(1)$ & $0.2206(2)$ \\
\hline S2 & $0.1896(1)$ & $0.2920(2)$ \\
\hline $\mathrm{C} 1$ & $0.0981(4)$ & $0.2819(6)$ \\
\hline N1 & $0.0064(3)$ & $0.3168(7)$ \\
\hline $\mathrm{C} 2$ & $-0.0727(6)$ & $0.331(1)$ \\
\hline C3 & $-0.1175(7)$ & $0.213(1)$ \\
\hline $\mathrm{C4}$ & $-0.0283(5)$ & $0.3505(9)$ \\
\hline C5 & $-0.0251(7)$ & $0.4846(9)$ \\
\hline N2 & $0.2872(4)$ & $0.0168(6)$ \\
\hline $\mathrm{O} 2$ & $0.2812(4)$ & $-0.0972(5)$ \\
\hline C6 & $0.3744(4)$ & $0.1234(6)$ \\
\hline O6 & $0.4155(4)$ & $0.0818(5)$ \\
\hline $\mathrm{C} 7$ & $0.4259(4)$ & $0.1882(7)$ \\
\hline O7 & $0.4966(3)$ & $0.1830(5)$ \\
\hline N8 & $0.3334(3)$ & $0.4017(5)$ \\
\hline C9 & $0.3223(4)$ & $0.4399(6)$ \\
\hline $\mathrm{C} 10$ & $0.3430(4)$ & $0.5662(7)$ \\
\hline C11 & $0.3754(4)$ & $0.6601(7)$ \\
\hline $\mathrm{C} 12$ & $0.3863(5)$ & $0.6217(7)$ \\
\hline $\mathrm{C13}$ & $0.3657(4)$ & $0.4931(7)$ \\
\hline
\end{tabular}

(e) $\left[\mathrm{Mo}\left(\mathrm{Et}_{2} \mathrm{NCS}_{2}\right)(\mathrm{CO})(\mathrm{NO})(\mathrm{dppe})\right](7)$

$\begin{array}{lrl}\text { Mo } & 0.08472(3) & 0.22115(2) \\ \text { P1 } & 0.2405(1) & 0.26312(5) \\ \text { P2 } & -0.0545(1) & 0.27263(6) \\ \text { S1 } & 0.0453(1) & 0.11248(6) \\ \text { S2 } & -0.1002(1) & 0.15963(6) \\ \text { O1 } & 0.2920(3) & 0.1590(2) \\ \text { O2 } & 0.0981(3) & 0.3436(2) \\ \text { N1 } & 0.2085(4) & 0.1820(2) \\ \text { N2 } & -0.1460(4) & 0.0424(2) \\ \text { C1 } & 0.0913(4) & 0.2974(2) \\ \text { C2 } & -0.0756(4) & 0.0983(2) \\ \text { C3 } & -0.2454(5) & 0.0281(3) \\ \text { C4 } & -0.3671(6) & 0.0256(4) \\ \text { C5 } & -0.1171(5) & -0.0089(3) \\ \text { C6 } & -0.1705(6) & 0.0059(3) \\ \text { C7 } & 0.1674(4) & 0.3144(2) \\ \text { C8 } & 0.0498(4) & 0.2799(2) \\ \text { C11 } & 0.3264(4) & 0.1995(2) \\ \text { C12 } & 0.3584(4) & 0.1405(2) \\ \text { C13 } & 0.4312(5) & 0.0938(3) \\ \text { C14 } & 0.4709(5) & 0.1040(3) \\ \text { C15 } & 0.4384(6) & 0.1615(3) \\ \text { C16 } & 0.3673(5) & 0.2089(3) \\ \text { C21 } & 0.3614(4) & 0.3191(2) \\ \text { C22 } & 0.4834(5) & 0.3020(2) \\ & & \end{array}$

$z \quad B_{\mathrm{eq}}{ }^{*}$

$\begin{aligned} 0.2329(4) & 4.2(3) \\ 0.2814(3) & 7.3(3) \\ 0.2265(4) & 4.0(3) \\ 0.2727(3) & 7.3(3) \\ 0.1725(3) & 3.0(2) \\ 0.1914(3) & 5.1(3) \\ 0.0514(3) & 3.2(2) \\ 0.1139(3) & 3.6(3) \\ 0.1782(5) & 6.3(5) \\ 0.0831(4) & 4.2(3) \\ 0.1311(5) & 5.6(5) \\ -0.0041(4) & 4.3(3) \\ -0.0427(5) & 6.3(4) \\ 0.0132(4) & 4.1(3) \\ -0.0555(4) & 6.5(4)\end{aligned}$

$0.07959(4) \quad 5.84(3)$

$-0.0008(1) \quad 6.51(9)$

$0.2091(1) \quad 7.0(1)$

$0.1162(4) \quad 6.6(3)$

$0.1348(4) \quad 9.5(4)$

$0.0439(8) \quad 17.2(8)$

$0.0547(7) \quad 18.1(8)$

$0.2434(6) \quad 10.9(5)$

$0.2488(7) \quad 14.9(7)$

$0.1183(4) \quad 8.6(4)$

$0.1443(4) \quad 12.4(4)$

$-0.0423(5) \quad 7.3(4)$

$-0.1113(4) \quad 11.3(3)$

$0.1636(4) \quad 6.9(3)$

$0.2145(3) \quad 10.1(3)$

$0.0278(3) \quad 5.4(4)$

$-0.0700(4) \quad 6.4(4)$

$-0.1031(4) \quad 7.5(4)$

$-0.0330(5) \quad 7.9(4)$

$0.0673(5) \quad 7.8(4)$

$0.0962(4) \quad 6.8(3)$

$0.06342(3) \quad 2.86(2)$

$0.20842(8) \quad 3.02(6)$

$0.14857(8) \quad 3.26(6)$

$0.13693(8) \quad 3.83(6)$

$-0.04975(8) \quad 3.90(6)$

$0.0112(2) \quad 5.1(2)$

$-0.0510(2) \quad 5.8(2)$

$0.0283(3) \quad 4.3(2)$

$0.0196(3) \quad 4.8(2)$

$-0.0051(3) \quad 2.7(2)$

$0.0341(3) \quad 3.7(3)$

$-0.0702(4) \quad 6.3(3)$

$-0.0633(5)$

$0.0941(5)$

$0.1679(5)$

$0.2729(3)$

$0.2712(3)$

$0.2926(3)$

$0.2600(3)$

$0.3221(4)$

$0.4164(4)$

$0.4499(4)$

$0.3886(3)$

$0.2012(3)$

$0.2354(4)$
Table 2 (continued)

\begin{tabular}{lrrrr}
\hline Atom & \multicolumn{1}{c}{$y$} & \multicolumn{1}{l}{$z$} & \multicolumn{1}{c}{$B_{\text {eq }}{ }^{a}$} \\
\hline C23 & $0.5722(4)$ & $0.3455(3)$ & $0.2262(4)$ & $5.6(3)$ \\
C24 & $0.5383(5)$ & $0.4059(3)$ & $0.1840(4)$ & $5.5(3)$ \\
C25 & $0.4174(5)$ & $0.4240(2)$ & $0.1501(4)$ & $5.7(3)$ \\
C26 & $0.3291(4)$ & $0.3810(2)$ & $0.1572(3)$ & $4.7(3)$ \\
C31 & $-0.1091(4)$ & $0.3592(2)$ & $0.1190(3)$ & $3.6(2)$ \\
C32 & $-0.1301(5)$ & $0.4018(3)$ & $0.1831(3)$ & $4.9(3)$ \\
C33 & $-0.1639(5)$ & $0.4682(3)$ & $0.1602(4)$ & $6.2(3)$ \\
C34 & $-0.1790(6)$ & $0.4914(3)$ & $0.0735(5)$ & $7.3(5)$ \\
C35 & $-0.1610(7)$ & $0.4500(4)$ & $0.0087(5)$ & $10.0(5)$ \\
C36 & $-0.1273(6)$ & $0.3833(3)$ & $0.0311(4)$ & $7.4(4)$ \\
C41 & $-0.1879(4)$ & $0.2280(2)$ & $0.1548(3)$ & $3.7(2)$ \\
C42 & $-0.2908(5)$ & $0.2218(3)$ & $0.0737(4)$ & $5.2(3)$ \\
C43 & $-0.3924(5)$ & $0.1861(3)$ & $0.0739(4)$ & $6.2(4)$ \\
C44 & $-0.3928(6)$ & $0.1577(3)$ & $0.1546(6)$ & $7.2(5)$ \\
C45 & $-0.2931(6)$ & $0.1642(3)$ & $0.2354(5)$ & $7.0(4)$ \\
C46 & $-0.1909(5)$ & $0.1993(3)$ & $0.2362(4)$ & $5.3(3)$
\end{tabular}

(f) $\left[\mathrm{Mo}\left(\mathrm{Et}_{2} \mathrm{NCS}_{2}\right)(\mathrm{CO})(\mathrm{NO})\left(\mathrm{PPh}_{3}\right)_{2}\right]$ (8)

\begin{tabular}{|c|c|c|c|c|}
\hline $\mathrm{Mo}$ & $0.05515(4)$ & $0.16060(2)$ & $0.20113(2)$ & $2.58(2)$ \\
\hline S1 & $0.2547(1)$ & $0.19704(7)$ & $0.28992(7)$ & $3.71(6)$ \\
\hline S2 & $0.1633(1)$ & $0.05317(7)$ & $0.27322(7)$ & $3.49(6)$ \\
\hline P1 & $-0.0458(1)$ & $0.15938(7)$ & $0.29858(7)$ & $2.81(6)$ \\
\hline P2 & $0.1808(1)$ & $0.15413(7)$ & $0.11556(7)$ & $2.86(6)$ \\
\hline $\mathrm{O} 1$ & $-0.1585(3)$ & $0.0983(2)$ & $0.0900(2)$ & $5.1(2)$ \\
\hline $\mathrm{O} 2$ & $-0.0368(3)$ & $0.3107(2)$ & $0.1567(2)$ & $6.2(3)$ \\
\hline N2 & $0.3671(5)$ & $0.0895(3)$ & $0.3682(3)$ & $10.4(4)$ \\
\hline N1 & $-0.0742(4)$ & $0.1219(2)$ & $0.1351(2)$ & $3.9(2)$ \\
\hline $\mathrm{C} 2$ & $-0.0007(4)$ & $0.2531(2)$ & $0.1724(3)$ & $3.1(2)$ \\
\hline $\mathrm{C} 3$ & $0.2744(4)$ & $0.1104(3)$ & $0.3161(3)$ & $4.7(3)$ \\
\hline $\mathrm{C} 4$ & $0.3691(6)$ & $0.0148(4)$ & $0.4070(4)$ & $9.9(5)$ \\
\hline $\mathrm{C} 5$ & $0.4289(7)$ & $-0.0248(5)$ & $0.3703(5)$ & $12.7(7)$ \\
\hline C6 & $0.4588(8)$ & $0.1423(4)$ & $0.4203(5)$ & $13.0(7)$ \\
\hline $\mathrm{C} 7$ & $0.5553(8)$ & $0.1335(5)$ & $0.4110(7)$ & $20(1)$ \\
\hline $\mathrm{C} 11$ & $0.0501(4)$ & $0.1900(3)$ & $0.3851(2)$ & $3.0(2)$ \\
\hline $\mathrm{C} 12$ & $0.0663(5)$ & $0.2619(3)$ & $0.3981(3)$ & $4.1(3)$ \\
\hline $\mathrm{C} 13$ & $0.1467(5)$ & $0.2875(3)$ & $0.4602(3)$ & $5.2(3)$ \\
\hline C14 & $0.2127(5)$ & $0.2402(3)$ & $0.5093(3)$ & $5.5(3)$ \\
\hline C15 & $0.2010(5)$ & $0.1693(3)$ & $0.4972(3)$ & $5.5(3)$ \\
\hline $\mathrm{C} 16$ & $0.1192(5)$ & $0.1436(3)$ & $0.4353(3)$ & $4.2(3)$ \\
\hline $\mathrm{C} 21$ & $-0.1052(4)$ & $0.0745(3)$ & $0.3182(2)$ & $3.0(2)$ \\
\hline $\mathrm{C} 22$ & $-0.1374(5)$ & $0.0628(3)$ & $0.3804(3)$ & $5.1(3)$ \\
\hline $\mathrm{C} 23$ & $-0.1892(6)$ & $0.0000(3)$ & $0.3910(3)$ & $6.5(4)$ \\
\hline $\mathrm{C} 24$ & $-0.2150(5)$ & $-0.0517(3)$ & $0.3392(3)$ & $5.6(3)$ \\
\hline $\mathrm{C} 25$ & $-0.1846(5)$ & $-0.0409(3)$ & $0.2774(3)$ & $4.9(3)$ \\
\hline $\mathrm{C} 26$ & $-0.1300(4)$ & $0.0215(3)$ & $0.2671(3)$ & $3.8(3)$ \\
\hline C31 & $-0.1800(4)$ & $0.2130(3)$ & $0.2840(2)$ & $3.0(2)$ \\
\hline C32 & $-0.2179(5)$ & $0.2402(3)$ & $0.3396(3)$ & $4.9(3)$ \\
\hline C33 & $-0.3251(5)$ & $0.2743(3)$ & $0.3267(3)$ & $5.8(4)$ \\
\hline C34 & $-0.3977(5)$ & $0.2818(3)$ & $0.2579(3)$ & $5.5(3)$ \\
\hline C35 & $-0.3633(5)$ & $0.2554(3)$ & $0.2025(3)$ & $5.1(3)$ \\
\hline C36 & $-0.2554(4)$ & $0.2208(3)$ & $0.2150(3)$ & $4.2(3)$ \\
\hline C41 & $0.2985(4)$ & $0.0878(3)$ & $0.1403(3)$ & $3.3(3)$ \\
\hline $\mathrm{C} 42$ & $0.4164(5)$ & $0.1064(3)$ & $0.1741(3)$ & $4.9(3)$ \\
\hline $\mathrm{C} 43$ & $0.5016(5)$ & $0.0545(4)$ & $0.1982(4)$ & $7.0(4)$ \\
\hline C44 & $0.4713(5)$ & $-0.0157(3)$ & $0.1897(4)$ & $7.0(4)$ \\
\hline C45 & $0.3551(5)$ & $-0.0349(3)$ & $0.1582(3)$ & $5.7(4)$ \\
\hline $\mathrm{C} 46$ & $0.2703(4)$ & $0.0166(3)$ & $0.1342(3)$ & $4.3(3)$ \\
\hline C51 & $0.2610(4)$ & $0.2341(3)$ & $0.1024(2)$ & $3.1(2)$ \\
\hline C52 & $0.2611(4)$ & $0.2954(3)$ & $0.1417(3)$ & $3.7(3)$ \\
\hline $\mathrm{C} 53$ & $0.3243(5)$ & $0.3552(3)$ & $0.1326(3)$ & $4.5(3)$ \\
\hline C54 & $0.3872(5)$ & $0.3540(3)$ & $0.0834(3)$ & $5.1(3)$ \\
\hline C55 & $0.3883(4)$ & $0.2941(3)$ & $0.0435(3)$ & $4.5(3)$ \\
\hline C56 & $0.3248(4)$ & $0.2343(3)$ & $0.0520(3)$ & $3.8(3)$ \\
\hline C61 & $0.0972(4)$ & $0.1332(2)$ & $0.0223(2)$ & $3.0(2)$ \\
\hline C62 & $0.1304(5)$ & $0.0830(3)$ & $-0.0195(3)$ & $4.9(3)$ \\
\hline
\end{tabular}


Table 2 (continued)

\begin{tabular}{|c|c|c|c|c|}
\hline Atom & $x$ & $y$ & $z$ & $B_{\mathrm{eq}}{ }^{\mathrm{a}}$ \\
\hline $\mathrm{C} 63$ & $0.0624(6)$ & $0.0718(3)$ & $-0.0900(3)$ & $5.8(4)$ \\
\hline $\mathrm{C} 64$ & $-0.0378(5)$ & $0.1105(3)$ & $-0.1200(3)$ & $5.3(3)$ \\
\hline $\mathrm{C} 65$ & $-0.0724(5)$ & $0.1607(3)$ & $-0.0789(3)$ & $5.0(3)$ \\
\hline C66 & $-0.0052(5)$ & $0.1715(3)$ & $-0.0081(3)$ & $4.1(3)$ \\
\hline $\mathrm{Cl}$ & $0.5658(4)$ & $0.9398(2)$ & $0.0345(3)$ & $29.2(5)$ \\
\hline $\mathrm{C} 8$ & $0.5866(9)$ & $1.0199(6)$ & $0.0260(7)$ & $5.8(7)$ \\
\hline \multicolumn{5}{|c|}{ (g) $\left[\mathrm{Mo}\left(\mathrm{Et}_{2} \mathrm{NCS}_{2}\right)(\mathrm{CO})(\mathrm{NO})\left\{\mathrm{P}(\mathrm{OMe})_{3}\right\}_{2}\right](10)$} \\
\hline Mo & $0.2141(1)$ & $0.15129(4)$ & $0.21547(9)$ & $3.06(5)$ \\
\hline P1 & $0.2454(4)$ & $0.1653(1)$ & $0.0036(3)$ & $3.4(2)$ \\
\hline P2 & $0.1790(5)$ & $0.1306(2)$ & $0.4247(3)$ & $4.5(2)$ \\
\hline S1 & $0.2311(4)$ & $0.0470(1)$ & $0.1683(3)$ & $4.6(2)$ \\
\hline S2 & $0.4911(4)$ & $0.1152(2)$ & $0.2638(3)$ & $5.3(2)$ \\
\hline $\mathrm{Cl}$ & $0.422(2)$ & $0.0520(6)$ & $0.216(1)$ & $5.8(8)$ \\
\hline N1 & $0.516(2)$ & $0.0073(5)$ & $0.213(2)$ & $10(1)$ \\
\hline $\mathrm{C} 2$ & $0.458(2)$ & $-0.0478(8)$ & $0.175(3)$ & $16(2)$ \\
\hline $\mathrm{C} 3$ & $0.408(3)$ & $-0.0834(9)$ & $0.265(3)$ & $22(3)$ \\
\hline $\mathrm{C} 4$ & $0.682(2)$ & $0.0101(9)$ & $0.249(3)$ & $15(2)$ \\
\hline $\mathrm{C} 5$ & $0.777(3)$ & $0.024(1)$ & $0.160(3)$ & $20(3)$ \\
\hline $\mathrm{N} 2$ & $0.005(1)$ & $0.1630(4)$ & $0.1795(9)$ & $4.7(6)$ \\
\hline $\mathrm{O} 2$ & $-0.134(1)$ & $0.1724(4)$ & $0.1600(8)$ & $6.3(6)$ \\
\hline $\mathrm{C} 6$ & $0.242(1)$ & $0.2274(5)$ & $0.2473(9)$ & $3.2(6)$ \\
\hline O6 & $0.261(1)$ & $0.2748(4)$ & $0.2610(8)$ & $6.4(6)$ \\
\hline O7 & $0.190(1)$ & $0.2264(3)$ & $-0.0369(7)$ & $4.8(5)$ \\
\hline $\mathrm{C} 7$ & $0.199(2)$ & $0.2460(6)$ & $-0.158(1)$ & $6.4(8)$ \\
\hline 08 & $0.407(1)$ & $0.1585(4)$ & $-0.0432(7)$ & $4.8(5)$ \\
\hline $\mathrm{C} 8$ & $0.531(2)$ & $0.1936(8)$ & $0.003(2)$ & $9(1)$ \\
\hline O9 & $0.160(1)$ & $0.1245(4)$ & $-0.0964(7)$ & $5.0(5)$ \\
\hline $\mathrm{C} 9$ & $0.004(2)$ & $0.1101(6)$ & $-0.096(1)$ & $5.9(8)$ \\
\hline O10 & $0.305(1)$ & $0.1492(4)$ & $0.5320(7)$ & $7.1(6)$ \\
\hline $\mathrm{C} 10$ & $0.398(2)$ & $0.1958(7)$ & $0.533(1)$ & $7.4(10)$ \\
\hline O11 & $0.023(1)$ & $0.1552(5)$ & $0.4519(8)$ & $7.3(6)$ \\
\hline C11 & $-0.035(2)$ & $0.1411(9)$ & $0.567(2$ & $12(1)$ \\
\hline 012 & $0.166(1)$ & $0.0672(4)$ & $0.4683(8)$ & $6.7(5)$ \\
\hline $\mathrm{C} 12^{\mathrm{b}}$ & $0.023(4)$ & $0.040(1)$ & $0.426(3)$ & $8.1(9)$ \\
\hline $\mathrm{C} 12^{\prime b}$ & $0.311(3)$ & $0.031(1)$ & $0.469(3)$ & $6.5(8)$ \\
\hline
\end{tabular}

${ }^{a} B_{\text {eq }}\left(\AA^{2}\right)$ is the mean of the principal axes of the thermal ellipsoid.

${ }^{b}$ Mean atoms with occupancy $=0.5$.

\section{Results and discussion}

Ten monomeric substitution products of $\mathrm{M}^{\prime}[\mathrm{Mo}-$ $\left.\left(\mathrm{Et}_{2} \mathrm{NCS}_{2}\right)(\mathrm{CO})_{2}(\mathrm{NO}) \mathrm{X}\right] \quad\left(\mathrm{M}^{++}=\mathrm{PPN}^{+}\right.$or $\mathrm{Et}_{4} \mathrm{~N}^{+}$; $\mathrm{X}^{-}=\mathrm{NCS}^{-}(1), \mathrm{NO}_{3}^{-}(2), \mathrm{N}_{3}^{-}(3), \mathrm{Br}^{-}$(4) or $\mathrm{Cl}^{-}$ (5)) and $\left[\mathrm{Mo}\left(\mathrm{Et}_{2} \mathrm{NCS}_{2}\right)(\mathrm{CO})(\mathrm{NO}) \mathrm{L}_{2}\right]\left(\mathrm{L}_{2}=\right.$ py and $\mathrm{CO}$ (6); dppe (7); $2 \mathrm{PPh}_{3}(8) ; 2 \mathrm{PMe}_{3}(9) ; 2 \mathrm{P}(\mathrm{OMe})_{3}(10)$ ) were obtained readily from the reactions of $\left[\mathrm{Mo}\left(\mathrm{Et}_{2} \mathrm{NCS}_{2}\right)(\mathrm{CO})_{2}(\mathrm{NO})\right]_{2}$, a dinuclear compound with two $\mu: \eta^{1}, \eta^{2}$-diethyldithiocarbamato bridging ligands [3], with suitable nucleophiles at $28{ }^{\circ} \mathrm{C}$. The facile preparation reflects the fragile nature of the sulfur bridges in the dimeric compound.

From the similarity in the IR spectral data of complexes 1-5 and the structures of 1,3 and 4 (Figs. 1-3), it is possible that all five compounds have a similar geometry with the nitrosyl group trans to $\mathrm{NCS}^{-}$in $\mathbf{1}$, $\mathrm{NO}_{3}{ }^{-}$in $2, \mathrm{~N}_{3}{ }^{-}$in $3, \mathrm{Br}^{-}$in 4 , and $\mathrm{Cl}^{-}$in 5. This indicates that the higher electrophilic nitrosyl, compared with $\mathrm{CO}$, prefers to occupy a position trans to a stronger electron donor, compared with $\eta^{2}-\mathrm{Et}_{2} \mathrm{NCS}_{2}{ }^{-}$in the
Table 3

Selected bond lengths $(\AA)$ and angles ( $\left(^{\circ}\right)$

$\begin{array}{lclc}\left.\left.\text { (a) [PPN][Mo( } \mathrm{Et}_{2} \mathrm{NCS}_{2}\right)(\mathrm{CO})_{2}(\mathrm{NO})(\mathrm{NCS})\right](1) & \\ \text { Mo-S(1) } & 2.543(2) & \mathrm{C}(1)-\mathrm{N}(1) & 1.156(8) \\ \text { Mo-S(2) } & 2.539(2) & \mathrm{C}(2)-\mathrm{N}(2) & 1.327(8) \\ \mathrm{Mo}-\mathrm{N}(1) & 2.180(5) & \mathrm{N}(2)-\mathrm{C}(3) & 1.467(8) \\ \text { Mo-N(3) } & 1.782(5) & \mathrm{N}(2)-\mathrm{C}(5) & 1.458(8) \\ \text { Mo-C(7) } & 2.001(7) & \mathrm{C}(3)-\mathrm{C}(4) & 1.50(1) \\ \mathrm{Mo}-\mathrm{C}(8) & 1.991(7) & \mathrm{C}(5)-\mathrm{C}(6) & 1.51(1) \\ \mathrm{S}(1)-\mathrm{C}(2) & 1.721(6) & \mathrm{N}(3)-\mathrm{O}(1) & 1.201(6) \\ \mathrm{S}(2)-\mathrm{C}(2) & 1.713(6) & \mathrm{C}(7)-\mathrm{O}(2) & 1.142(9) \\ \mathrm{S}(3)-\mathrm{C}(1) & 1.618(6) & \mathrm{C}(8)-\mathrm{O}(3) & 1.143(8) \\ \mathrm{S}(1)-\mathrm{Mo}-\mathrm{S}(2) & 69.27(5) & \mathrm{S}(1)-\mathrm{Mo}-\mathrm{C}(8) & 100.6(2) \\ \mathrm{N}(1)-\mathrm{Mo}-\mathrm{N}(3) & 173.0(2) & \mathrm{S}(2)-\mathrm{Mo}-\mathrm{C}(7) & 99.7(2) \\ \mathrm{C}(7)-\mathrm{Mo}-\mathrm{C}(8) & 90.3(3) & \mathrm{C}(3)-\mathrm{N}(2)-\mathrm{C}(5) & 116.3(5) \\ \text { S(3)-C(1)-N(1) } & 179.1(5) & \mathrm{Mo}-\mathrm{N}(3)-\mathrm{O}(1) & 175.1(4) \\ \mathrm{Mo}-\mathrm{N}(1)-\mathrm{C}(1) & 172.8(5) & \mathrm{Mo}-\mathrm{C}(7)-\mathrm{O}(2) & 174.7(5) \\ \mathrm{S}(1)-\mathrm{C}(2)-\mathrm{S}(2) & 114.5(3) & \mathrm{Mo}-\mathrm{C}(8)-\mathrm{O}(3) & 178.3(5)\end{array}$

(b) $[\mathrm{PPN}]\left[\mathrm{Mo}\left(\mathrm{Et}_{2} \mathrm{NCS}_{2}\right)(\mathrm{CO})_{2}(\mathrm{NO})\left(\eta^{1}-\mathrm{N}_{3}\right)\right](3)$

$\begin{array}{lclr}\text { Mo-S(1) } & 2.524(2) & \mathrm{N}(2)-\mathrm{N}(3) & 1.152(8) \\ \mathrm{Mo}-\mathrm{S}(2) & 2.526(2) & \mathrm{N}(4)-\mathrm{O}(4) & 1.193(6) \\ \mathrm{Mo}-\mathrm{N}(1) & 2.195(5) & \mathrm{C}(1)-\mathrm{O}(1) & 1.142(8) \\ \mathrm{Mo}-\mathrm{N}(4) & 1.795(5) & \mathrm{C}(2)-\mathrm{O}(2) & 1.143(8) \\ \mathrm{Mo}-\mathrm{C}(1) & 2.002(7) & \mathrm{C}(3)-\mathrm{N}(5) & 1.335(7) \\ \mathrm{Mo}-\mathrm{C}(2) & 2.005(7) & \mathrm{N}(5)-\mathrm{C}(4) & 1.475(8) \\ \mathrm{S}(1)-\mathrm{C}(3) & 1.707(6) & \mathrm{N}(5)-\mathrm{C}(6) & 1.460(8) \\ \mathrm{S}(2)-\mathrm{C}(3) & 1.710(6) & \mathrm{C}(4)-\mathrm{C}(5) & 1.50(1) \\ \mathrm{N}(1)-\mathrm{N}(2) & 1.160(8) & \mathrm{C}(6)-\mathrm{C}(7) & 1.50(1) \\ \mathrm{S}(1)-\mathrm{Mo}-\mathrm{S}(2) & 70.04(5) & \mathrm{N}(1)-\mathrm{N}(2)-\mathrm{N}(3) & 177.3(7) \\ \mathrm{S}(1)-\mathrm{Mo}-\mathrm{C}(1) & 98.8(2) & \mathrm{Mo}-\mathrm{N}(4)-\mathrm{O}(4) & 177.5(4) \\ \mathrm{S}(2)-\mathrm{Mo}-\mathrm{C}(2) & 96.1(2) & \mathrm{Mo}-\mathrm{C}(1)-\mathrm{O}(1) & 176.4(5) \\ \mathrm{N}(1)-\mathrm{Mo}-\mathrm{N}(4) & 173.4(2) & \mathrm{Mo}-\mathrm{C}(2)-\mathrm{O}(2) & 176.9(6) \\ \mathrm{C}(1)-\mathrm{Mo}-\mathrm{C}(2) & 94.5(3) & \mathrm{S}(1)-\mathrm{C}(3)-\mathrm{S}(2) & 116.0(3) \\ \mathrm{Mo}-\mathrm{N}(1)-\mathrm{N}(2) & 129.2(4) & \mathrm{C}(4)-\mathrm{N}(5)-\mathrm{C}(6) & 116.6(4)\end{array}$

(c) $\left[\mathrm{Et}_{4} \mathrm{~N}\right]\left[\mathrm{Mo}\left(\mathrm{Et}_{2} \mathrm{NCS}_{2}\right)(\mathrm{CO})_{2}(\mathrm{NO})(\mathrm{Br})\right](4)$

$\begin{array}{lclc}\text { Mo-Br } & 2.659(1) & \mathrm{C}(3)-\mathrm{C}(4) & 1.52(1) \\ \mathrm{Mo}-\mathrm{S}(1) & 2.528(2) & \mathrm{C}(5)-\mathrm{O}(5) & 1.146(9) \\ \mathrm{Mo}-\mathrm{S}(2) & 2.550(2) & \mathrm{C}(6)-\mathrm{O}(6) & 1.137(9) \\ \mathrm{Mo}-\mathrm{C}(5) & 2.004(8) & \mathrm{N}(7)-\mathrm{O}(7) & 1.181(7) \\ \mathrm{Mo}-\mathrm{C}(6) & 2.004(8) & \mathrm{C}-\mathrm{N}(1) & 1.328(8) \\ \mathrm{Mo}-\mathrm{N}(7) & 1.786(5) & \mathrm{N}(1)-\mathrm{C}(1) & 1.47(1) \\ \mathrm{S}(1)-\mathrm{C} & 1.720(6) & \mathrm{N}(1)-\mathrm{C}(3) & 1.49(1) \\ \text { S(2)-C } & 1.708(6) & \mathrm{C}(1)-\mathrm{C}(2) & 1.46(1) \\ \mathrm{C}(1)-\mathrm{N}(1)-\mathrm{C}(3) & 115.5(5) & \mathrm{Br}-\mathrm{Mo}-\mathrm{N}(7) & 172.4(1) \\ \mathrm{S}(1)-\mathrm{Mo}-\mathrm{S}(2) & 69.61(6) & \mathrm{N}(1)-\mathrm{C}(3)-\mathrm{C}(4) & 109.6(7) \\ \mathrm{S}(1)-\mathrm{Mo}-\mathrm{C}(5) & 98.9(2) & \mathrm{Mo}-\mathrm{C}(5)-\mathrm{O}(5) & 177.6(6) \\ \mathrm{Mo}-\mathrm{C}(6)-\mathrm{O}(6) & 176.3(7) & \mathrm{C}(5)-\mathrm{Mo}-\mathrm{C}(6) & 88.2(3) \\ \mathrm{Mo}-\mathrm{N}(7)-\mathrm{O}(7) & 175.8(4) & \mathrm{S}(1)-\mathrm{C}-\mathrm{S}(2) & 115.4(3)\end{array}$

$\mathrm{S}(2)-\mathrm{Mo}-\mathrm{C}(6)$

$102.7(2)$

(d) $\left[\mathrm{Mo}\left(\mathrm{Et}_{2} \mathrm{NCS}_{2}\right)(\mathrm{CO})_{2}(\mathrm{NO}) \mathrm{py}\right](6)$

$\begin{array}{lcll}\text { Mo-S }(1) & 2.523(2) & \mathrm{C}(2)-\mathrm{C}(3) & 1.34(2) \\ \text { Mo-S(2) } & 2.518(2) & \mathrm{C}(4)-\mathrm{C}(5) & 1.35(1) \\ \text { Mo-N(2) } & 1.813(6) & \mathrm{N}(2)-\mathrm{O}(2) & 1.196(8) \\ \text { Mo-C(6) } & 1.993(6) & \mathrm{C}(6)-\mathrm{O}(6) & 1.144(7) \\ \text { Mo-C(7) } & 1.968(6) & \mathrm{C}(7)-\mathrm{O}(7) & 1.146(7) \\ \mathrm{Mo}-\mathrm{N}(8) & 2.280(5) & \mathrm{N}(8)-\mathrm{C}(9) & 1.329(7) \\ \mathrm{S}(1)-\mathrm{C}(1) & 1.709(6) & \mathrm{N}(8)-\mathrm{C}(13) & 1.340(7) \\ \mathrm{S}(2)-\mathrm{C}(1) & 1.703(6) & \mathrm{C}(9)-\mathrm{C}(10) & 1.369(9) \\ \mathrm{C}(1)-\mathrm{N}(1) & 1.315(7) & \mathrm{C}(10)-\mathrm{C}(11) & 1.371(9) \\ \mathrm{N}(1)-\mathrm{C}(2) & 1.57(1) & \mathrm{C}(11)-\mathrm{C}(12) & 1.360(9) \\ \mathrm{N}(1)-\mathrm{C}(4) & 1.535(9) \mathrm{C}(12)-\mathrm{C}(13) & 1.374(9) \\ \mathrm{S}(1)-\mathrm{Mo}-\mathrm{S}(2) & 70.25(5) & \mathrm{S}(1)-\mathrm{Mo}-\mathrm{C}(6) & 100.2(2) \\ \mathrm{S}(2)-\mathrm{Mo}-\mathrm{C}(7) & 98.7(2) & \mathrm{Mo}-\mathrm{N}(2)-\mathrm{O}(2) & 177.5(5)\end{array}$

(continued) 
Table 3 (continued)

\begin{tabular}{lrll}
\hline N(2)-Mo-N(8) & $175.5(2)$ & Mo-N(8)-C(13) & $120.3(4)$ \\
C(6)-Mo-C(7) & $90.6(2)$ & S(1)-C(1)-S(2) & $116.4(3)$ \\
Mo-C(6)-O(6) & $177.9(6)$ & Mo-C(7)-O(7) & $177.2(6)$ \\
Mo-N(8)-C(9) & $122.3(4)$ & C(2)-N(1)-C(4) & $116.7(5)$
\end{tabular}

(e) $\left[\mathrm{Mo}\left(\mathrm{Et}_{2} \mathrm{NCS}_{2}\right)(\mathrm{CO})(\mathrm{NO})(\mathrm{dppe})\right](7)$

$\begin{array}{lclr}\text { Mo-P(1) } & 2.444(1) & \mathrm{P}(2)-\mathrm{C}(41) & 1.820(5) \\ \text { Mo-P(2) } & 2.620(1) & \mathrm{S}(1)-\mathrm{C}(2) & 1.709(5) \\ \text { Mo-S(1) } & 2.549(1) & \mathrm{S}(2)-\mathrm{C}(2) & 1.714(5) \\ \text { Mo-S(2) } & 2.535(1) & \mathrm{O}(1)-\mathrm{N}(1) & 1.185(5) \\ \text { Mo-N(1) } & 1.876(4) & \mathrm{O}(2)-\mathrm{C}(1) & 1.175(5) \\ \text { Mo-C(1) } & 1.857(4) & \mathrm{N}(2)-\mathrm{C}(2) & 1.350(6) \\ \mathrm{P}(1)-\mathrm{C}(7) & 1.824(4) & \mathrm{N}(2)-\mathrm{C}(3) & 1.466(7) \\ \mathrm{P}(1)-\mathrm{C}(11) & 1.821(4) & \mathrm{N}(2)-\mathrm{C}(5) & 1.472(7) \\ \mathrm{P}(1)-\mathrm{C}(21) & 1.828(4) & \mathrm{C}(3)-\mathrm{C}(4) & 1.458(9) \\ \mathrm{P}(2)-\mathrm{C}(8) & 1.829(4) & \mathrm{C}(5)-\mathrm{C}(6) & 1.50(1) \\ \mathrm{P}(2)-\mathrm{C}(31) & 1.835(5) & \mathrm{C}(7)-\mathrm{C}(8) & 1.521(7) \\ \mathrm{P}(1)-\mathrm{Mo}-\mathrm{P}(2) & 79.00(4) & \mathrm{P}(1)-\mathrm{C}(7)-\mathrm{C}(8) & 109.3(3) \\ \mathrm{P}(1)-\mathrm{Mo}-\mathrm{N}(1) & 90.2(1) & \mathrm{P}(2)-\mathrm{C}(8)-\mathrm{C}(7) & 108.4(3) \\ \text { S(1)-Mo-S(2) } & 69.31(4) & \mathrm{S}(1)-\mathrm{Mo}-\mathrm{C}(1) & 171.3(1) \\ \text { Mo-P(1)-C(7) } & 109.8(1) & \mathrm{Mo}-\mathrm{P}(2)-\mathrm{C}(41) & 121.8(2) \\ \text { Mo-P(1)-C(11) } & 116.0(1) & \mathrm{Mo}-\mathrm{N}(1)-\mathrm{O}(1) & 175.8(3) \\ \text { Mo-P(1)-C(21) } & 119.3(1) & \mathrm{C}(3)-\mathrm{N}(2)-\mathrm{C}(5) & 118.0(4) \\ \text { Mo-P(2)-C(8) } & 103.6(1) & \mathrm{Mo}-\mathrm{C}(1)-\mathrm{O}(2) & 176.6(4) \\ \text { Mo-P(2)-C(31) } & 117.8(2) & \mathrm{S}(1)-\mathrm{C}(2)-\mathrm{S}(2) & 115.2(3) \\ \mathrm{P}(2)-\mathrm{Mo}-\mathrm{S}(2) & 90.02(4) & & \\ \mathrm{P}(2)-\mathrm{Mo}-\mathrm{P}(1)-\mathrm{C}(7) & -9.4(1) & \mathrm{Mo}-\mathrm{P}(1)-\mathrm{C}(7)-\mathrm{C}(8) & 42.8(2) \\ \mathrm{P}(1)-\mathrm{C}(7)-\mathrm{C}(8)-\mathrm{P}(2) & -61.2(2) & \mathrm{P}(1)-\mathrm{Mo}-\mathrm{P}(2)-\mathrm{C}(8) & -18.0(1) \\ \text { Mo-P(2)-C(8)-C(7) } & 49.3(2) & & \end{array}$

(f) $\left[\mathrm{Mo}\left(\mathrm{Et}_{2} \mathrm{NCS}_{2}\right)(\mathrm{CO})(\mathrm{NO})\left(\mathrm{PPh}_{3}\right)_{2}\right](8)$

$\begin{array}{llll}\text { Mo-S }(1) & 2.565(2) & \mathrm{P}(2)-\mathrm{C}(41) & 1.822(5) \\ \mathrm{Mo}-\mathrm{S}(2) & 2.576(1) & \mathrm{P}(2)-\mathrm{C}(51) & 1.837(5) \\ \mathrm{Mo}-\mathrm{P}(1) & 2.515(1) & \mathrm{P}(2)-\mathrm{C}(61) & 1.832(5) \\ \mathrm{Mo}-\mathrm{P}(2) & 2.533(1) & \mathrm{O}(1)-\mathrm{N}(1) & 1.199(5) \\ \mathrm{Mo}-\mathrm{N}(1) & 1.830(4) & \mathrm{O}(2)-\mathrm{C}(2) & 1.173(6) \\ \mathrm{Mo}-\mathrm{C}(2) & 1.889(5) & \mathrm{N}(2)-\mathrm{C}(3) & 1.311(7) \\ \mathrm{S}(1)-\mathrm{C}(3) & 1.707(5) & \mathrm{N}(2)-\mathrm{C}(4) & 1.594(9) \\ \mathrm{S}(2)-\mathrm{C}(3) & 1.710(5) & \mathrm{N}(2)-\mathrm{C}(6) & 1.59(1) \\ \mathrm{P}(1)-\mathrm{C}(11) & 1.821(5) & \mathrm{C}(4)-\mathrm{C}(5) & 1.36(1) \\ \mathrm{P}(1)-\mathrm{C}(21) & 1.831(5) & \mathrm{C}(6)-\mathrm{C}(7) & 1.21(1)\end{array}$

$P(1)-C(31)$

$\mathrm{S}(1)-\mathrm{Mo}-\mathrm{S}(2)$

$\mathrm{S}(1)-\mathrm{Mo}-\mathrm{C}(2)$

$\mathrm{S}(2)-\mathrm{Mo}-\mathrm{N}(1)$

$\mathrm{P}(1)-\mathrm{Mo}-\mathrm{P}(2)$

$\mathrm{N}(1)-\mathrm{Mo}-\mathrm{C}(2)$

Mo-P(1)-C(11)

Mo-P(1)-C(21)

$1.826(5)$

68.70(5) $\quad \mathrm{Mo}-\mathrm{P}(2)-\mathrm{C}(41) \quad 113.9(2)$

97.0(1) Mo-P(2)-C(51) 118.4(2)

104.1(1) Mo-P(2)-C(61) 114.4(2)

$172.17(4) \quad \mathrm{C}(4)-\mathrm{N}(2)-\mathrm{C}(6) \quad 109.8(5)$

90.9(2) Mo-N(1)-O(1) 177.7(4)

$113.0(2) \quad \mathrm{Mo}-\mathrm{C}(2)-\mathrm{O}(2) \quad 177.5(4)$

$117.4(2) \quad \mathrm{S}(1)-\mathrm{C}(3)-\mathrm{S}(2) \quad 116.2(3)$

Mo-P(1)-C(31) 117.2(2)

(g) $\left[\mathrm{Mo}\left(\mathrm{Et}_{2} \mathrm{NCS}_{2}\right)(\mathrm{CO})(\mathrm{NO})\left\{\mathrm{P}(\mathrm{OMe})_{3}\right\}_{2}\right](10)$

$\begin{array}{lll}\text { Mo-P(1) } & 2.469(3) & \mathrm{C}(1)-\mathrm{N}(1) \\ \mathrm{Mo-P}(2) & 2.475(4) & \mathrm{N}(1)-\mathrm{C}(2) \\ \mathrm{Mo}-\mathrm{S}(1) & 2.565(4) & \mathrm{N}(1)-\mathrm{C}(4) \\ \mathrm{Mo}-\mathrm{S}(2) & 2.559(4) & \mathrm{C}(2)-\mathrm{C}(3) \\ \mathrm{Mo-N}(2) & 1.84(1) & \mathrm{C}(4)-\mathrm{C}(5) \\ \mathrm{Mo}-\mathrm{C}(6) & 1.87(1) & \mathrm{N}(2)-\mathrm{O}(2) \\ \mathrm{P}(1)-\mathrm{O}(7) & 1.592(8) & \mathrm{C}(6)-\mathrm{O}(6) \\ \mathrm{P}(1)-\mathrm{O}(8) & 1.580(9) & \mathrm{O}(7)-\mathrm{C}(7) \\ \mathrm{P}(1)-\mathrm{O}(9) & 1.602(9) & \mathrm{O}(8)-\mathrm{C}(8) \\ \mathrm{P}(2)-\mathrm{O}(10) & 1.59(1) & \mathrm{O}(9)-\mathrm{C}(9) \\ \mathrm{P}(2)-\mathrm{O}(11) & 1.56(1) & \mathrm{O}(10)-\mathrm{C}(10) \\ \mathrm{P}(2)-\mathrm{O}(12) & 1.61(1) & \mathrm{O}(11)-\mathrm{C}(11) \\ \mathrm{S}(1)-\mathrm{C}(1) & 1.68(1) & \mathrm{O}(12)-\mathrm{C}(12)\end{array}$

$1.36(2)$

$1.46(2)$

$1.45(2)$

$1.44(4)$

$1.42(4)$

$1.22(2)$

$1.16(1)$

$1.46(1)$

$1.42(2)$

$1.40(2)$

$1.38(2)$

$1.46(2)$

$1.42(4)$

(continued)
Table 3 (continued)

\begin{tabular}{lrll}
\hline $\mathrm{S}(2)-\mathrm{C}(1)$ & $1.69(2)$ & $\mathrm{O}(12)-\mathrm{C}\left(12^{\prime}\right)$ & $1.54(3)$ \\
$\mathrm{P}(1)-\mathrm{Mo}-\mathrm{P}(2)$ & $176.2(1)$ & $\mathrm{S}(1)-\mathrm{C}(1)-\mathrm{S}(2)$ & $117.6(8)$ \\
$\mathrm{S}(1)-\mathrm{Mo}-\mathrm{N}(2)$ & $100.7(3)$ & $\mathrm{C}(2)-\mathrm{N}(1)-\mathrm{C}(4)$ & $114.9(14)$ \\
$\mathrm{S}(2)-\mathrm{Mo}-\mathrm{C}(6)$ & $101.2(4)$ & $\mathrm{Mo}-\mathrm{N}(2)-\mathrm{O}(2)$ & $177.1(9)$ \\
$\mathrm{N}(2)-\mathrm{Mo}-\mathrm{C}(6)$ & $89.6(5)$ & $\mathrm{Mo}-\mathrm{C}(6)-\mathrm{O}(6)$ & $176.5(10)$ \\
Mo-P(1)-O(7) & $109.6(3)$ & $\mathrm{Mo}-\mathrm{P}(2)-\mathrm{O}(10)$ & $120.8(4)$ \\
Mo-P(1)-O(8) & $122.0(3)$ & Mo-P(2)-O(11) & $109.7(4)$ \\
Mo-P(1)-O(9) & $120.0(3)$ & $\mathrm{Mo}-\mathrm{P}(2)-\mathrm{O}(12)$ & $120.4(4)$ \\
S(1)-Mo-S(2) & $68.6(1)$ & & \\
\hline
\end{tabular}

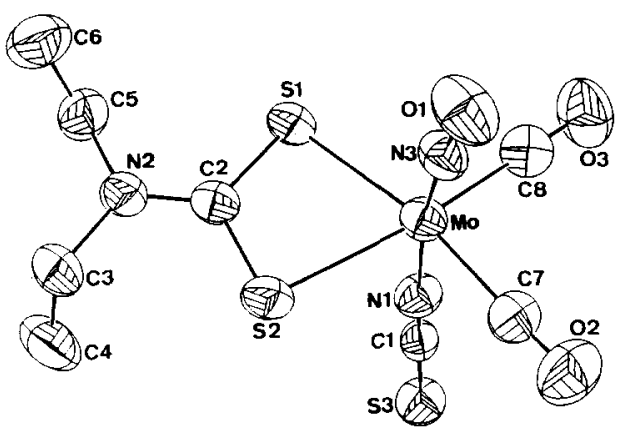

Fig. 1. ORTEP plot of $\left[\mathrm{Mo}\left(\mathrm{Et}_{2} \mathrm{NCS}_{2}\right)(\mathrm{CO})_{2}(\mathrm{NO})(\mathrm{NCS})\right]^{-}\left(1^{-}\right)$.

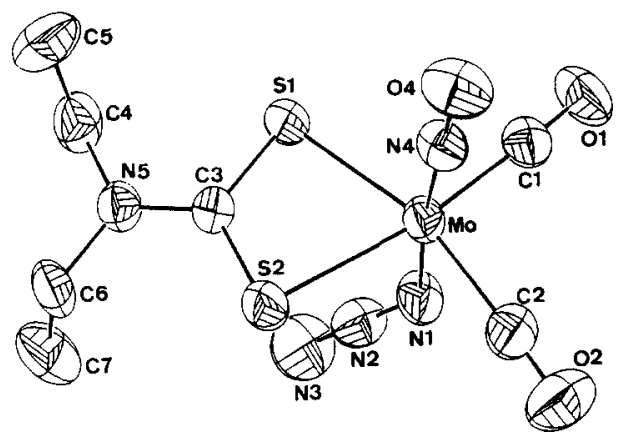

Fig. 2. ORTEP plot of $\left[\mathrm{Mo}\left(\mathrm{Et}_{2} \mathrm{NCS}_{2}\right)(\mathrm{CO})_{2}(\mathrm{NO})\left(\eta^{1}-\mathrm{N}_{3}\right)\right]^{-}\left(3^{-}\right)$.

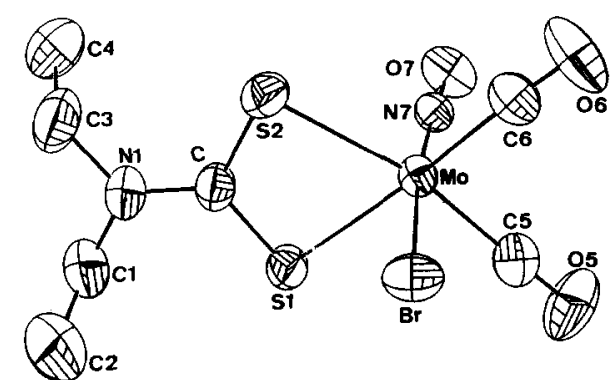

Fig. 3. ORTEP plot of $\left[\mathrm{Mo}\left(\mathrm{Et}_{2} \mathrm{NCS}_{2}\right)(\mathrm{CO})_{2}(\mathrm{NO})(\mathrm{Br})\right]^{-}\left(4^{-}\right)$.

compounds. Apparently this unique nitrosyl position can help to dissipate any accumulated charge density after coordination of a strong electron donor through a metal-to-nitrosyl backbonding.

The structure of 6 was also found to have py trans to NO (Fig. 4). However, there are two nitrosyl frequencies observed both in solution and in the solid state, indicating that 6 may have two isomers. Only 


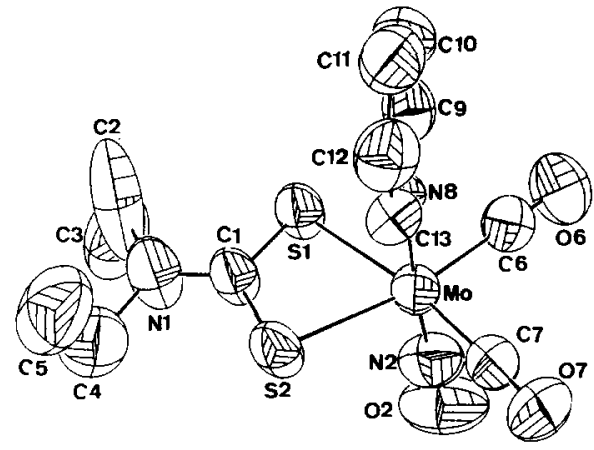

Fig. 4. ORTEP plot of $\left[\mathrm{Mo}\left(\mathrm{Et}_{2} \mathrm{NCS}_{2}\right)(\mathrm{CO})_{2}(\mathrm{NO})\right.$ py $](6)$.

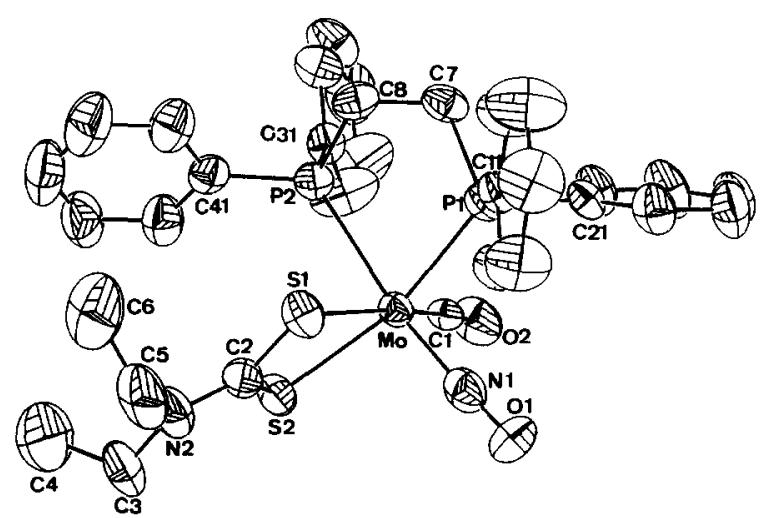

Fig. 5. ORTEP plot of $\left[\mathrm{Mo}\left(\mathrm{Et}_{2} \mathrm{NCS}_{2}\right)(\mathrm{CO})(\mathrm{NO})(\mathrm{dppe})\right]$ (7). Carbon atoms of $C(7), C(11)$ and $C(21)$ are connected to $P(1)$ and those of $C(8), C(31)$ and $C(41)$ are connected to $P(2)$.

two rather than four carbonyl stretching bands observed for 6 may be due to the accidental overlap of the close bands or the poor resolution of the IR machine used. This may also explain why only one $\mathrm{CN}$ stretching band for the diethyldithiocarbamato ligands in the two isomers was observed. The isomer shown in Fig. 4 may represent the one more easily crystallized from the solution, while the other is believed to have a different disposition of py, possibly py trans to CO.

Although only one ${ }^{31} \mathrm{P}$ singlet is observed for 8,9 and 10 at $43.05,-6.62$ and $159.30 \mathrm{ppm}$, respectively, there are four singlets observed at $67.76,64.22,41.10$ and $23.07 \mathrm{ppm}$ for 7. From the spectral data of 7-10 and the structures of 7, 8 and 10 (Figs. 5-7), we believe that the structure of 9 is similar to that of 8 or $\mathbf{1 0}$, with the two bulky phosphine ligands trans to each other, apparently to minimize the steric hindrance between the ligands, and that like 6, two isomeric structures are also present for 7. However, the two probable isomeric structures may involve either different orientations of phenyl groups with respect to the fivemembered ring formed by dppe and Mo, or may have different dispositions of the nitrosyl and carbonyl groups. By comparing the torsional angles in a range between -61.2 and $49.3^{\circ}$ (Table 3 ), the five-membered ring has close to a skew-boat geometry [7]. Based on the ${ }^{31} \mathrm{P}$

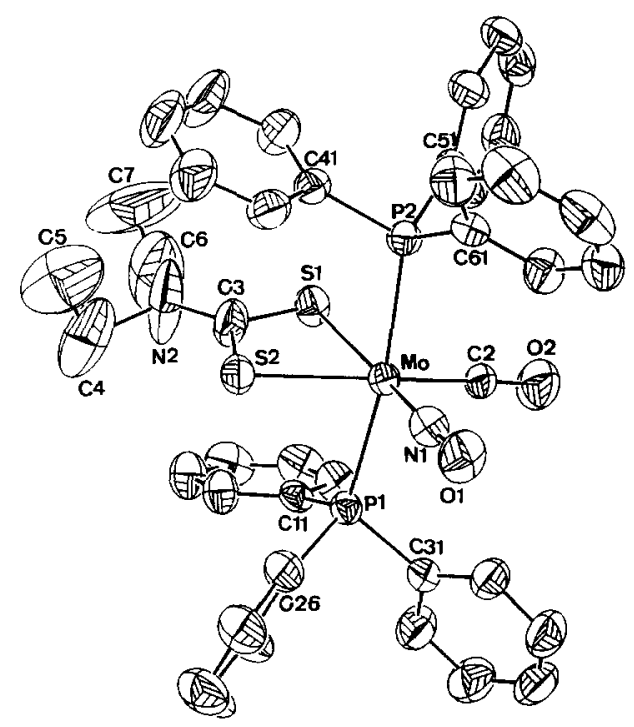

Fig. 6. ORTEP plot of $\left[\mathrm{Mo}\left(\mathrm{Et}_{2} \mathrm{NCS}_{2}\right)(\mathrm{CO})(\mathrm{NO})\left(\mathrm{PPh}_{3}\right)_{2}\right](8)$. Carbon atoms of $C(11), C(21)$ and $C(31)$ are connected to $P(1)$ and those of $C(41), C(51)$ and $C(61)$ are connected to $P(2) ; C(21)$ is below $C(26)$.

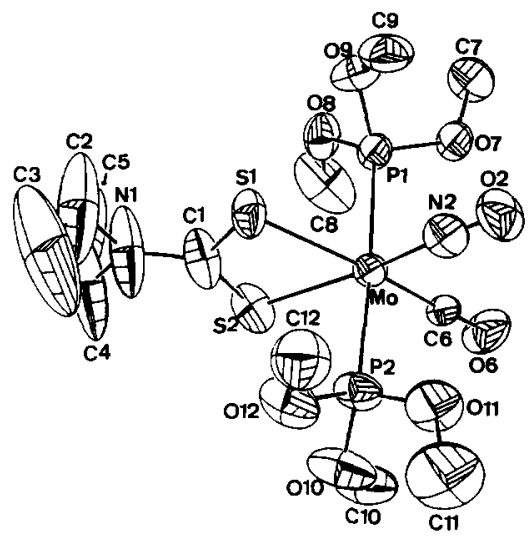

Fig. 7. ORTEP plot of $\left[\mathrm{Mo}\left(\mathrm{Et}_{2} \mathrm{NCS}_{2}\right)(\mathrm{CO})(\mathrm{NO})\left\{\mathrm{P}(\mathrm{OMe})_{3}\right\}_{2}\right](\mathbf{1 0})$.

NMR chemical shifts and theory of Letcher and Van Wazer [8], structures with different arrangements of $\mathrm{NO}$ and $\mathrm{CO}$ appear more probable. According to this theory, the singlet at 67.76 (or 64.22) and the one at $41.10 \mathrm{ppm}$ are assigned to one isomer, with this signal for the phosphorus atom trans to NO, and the singlet at 64.22 (or 67.76) and the one at $23.07 \mathrm{ppm}$ are assigned to the other isomer, with this signal for the phosphorus atom also trans to NO. The two possible arrangements for 6 and 7, we believe, may reflect the weaker $\sigma$-donicity of py and phosphine ligands than the uninegative anionic ligand such as $\mathrm{NCS}^{-}$in $\mathbf{1}$, $\mathrm{NO}_{3}^{-}$in $2, \mathrm{~N}_{3}^{-}$in $3, \mathrm{Br}^{-}$in 4 , and $\mathrm{Cl}^{-}$in 5 . It is worth remembering that the different preferred geometries of $\left[\mathrm{Mo}(\mathrm{L}-\mathrm{L})(\mathrm{CO})_{2}\left(\eta^{3}\right.\right.$-allyl)X] $\left(\mathrm{L}-\mathrm{L}=2,2^{\prime}\right.$-bipyridine (bpy) and dppe; $\mathrm{X}^{-}=$halide anion) were previously attributed to the $\sigma$-donicity difference between the two ligands [9].

The metal atom in each of the seven X-ray structures is six-coordinate. The angles, formed by two ligands and the metal atom are in the range $68.6-104.1^{\circ}$ (Table 
Table 4

Relevant parameters of molybdenum( 0 ) compounds containing dithiocarbamato ligands

\begin{tabular}{|c|c|c|c|c|c|c|}
\hline Compound & $\begin{array}{l}\text { MO-S } \\
(\AA)\end{array}$ & C-S $(\AA)$ & $\mathrm{C}-\mathrm{N}(\AA)$ & $S \cdots S(\AA)$ & $\angle S C S\left(^{\circ}\right)$ & Ref. \\
\hline$[\mathrm{PPN}]\left[\mathrm{Mo}\left(\mathrm{C}_{4} \mathrm{H}_{8} \mathrm{NCS}_{2}\right)(\mathrm{CO})_{4}\right]^{n}$ & $\begin{array}{l}2.600(1) \\
2.631(1)\end{array}$ & $\begin{array}{l}1.704(4) \\
1.716(4)\end{array}$ & $1.319(5)$ & $2.908(2)$ & $116.4(2)$ & [12] \\
\hline$[\mathrm{PPN}]\left[\mathrm{Mo}\left(\mathrm{C}_{5} \mathrm{H}_{10} \mathrm{NCS}_{2}\right)(\mathrm{CO})_{4}\right]^{\mathrm{b}}$ & $\begin{array}{l}2.598(5) \\
2.597(5)\end{array}$ & $\begin{array}{l}1.72(2) \\
1.69(2)\end{array}$ & $1.34(2)$ & $2.892(6)$ & $116.1(10)$ & {$[12]$} \\
\hline$[\mathrm{PPN}]\left[\mathrm{Mo}\left(\mathrm{Et}_{2} \mathrm{NCS}_{2}\right)(\mathrm{CO})_{4}\right]^{\mathrm{c}}$ & $2.596(1)$ & $1.704(3)$ & $1.343(7)$ & $2.90(1)$ & $116.4(3)$ & [11] \\
\hline$[\mathrm{PPN}]\left[\mathrm{Mo}\left(\mathrm{Et}_{2} \mathrm{NCS}_{2}\right)(\mathrm{CO})_{2}(\mathrm{NO})(\mathrm{NCS})\right]$ & $\begin{array}{l}2.543(2) \\
2.549(2)\end{array}$ & $\begin{array}{l}1.721(6) \\
1.713(6)\end{array}$ & $1.327(8)$ & $2.889(2)$ & $114.5(3)$ & this work \\
\hline$[\mathrm{PPN}]\left[\mathrm{Mo}\left(\mathrm{Et}_{2} \mathrm{NCS}_{2}\right)(\mathrm{CO})_{2}(\mathrm{NO})\left(\eta^{1} \cdot \mathrm{N}_{3}\right)\right]$ & $\begin{array}{l}2.524(2) \\
2.526(2)\end{array}$ & $\begin{array}{l}1.707(6) \\
1.710(6)\end{array}$ & $1.335(7)$ & $2.898(2)$ & $116.0(3)$ & this work \\
\hline$\left[\mathrm{Et}_{4} \mathrm{~N}\right]\left[\mathrm{Mo}\left(\mathrm{Et}_{2} \mathrm{NCS}_{2}\right)(\mathrm{CO})_{2}(\mathrm{NO})(\mathrm{Br})\right]$ & $\begin{array}{l}2.528(2) \\
2.550(2)\end{array}$ & $\begin{array}{l}1.720(6) \\
1.708(6)\end{array}$ & $1.328(8)$ & $2.898(3)$ & $115.4(3)$ & this work \\
\hline$\left[\mathrm{Mo}\left(\mathrm{Et}_{2} \mathrm{NCS}_{2}\right)(\mathrm{CO})_{2}(\mathrm{NO})(\mathrm{py})\right]$ & $\begin{array}{l}2.523(2) \\
2.518(2)\end{array}$ & $\begin{array}{l}1.709(6) \\
1.703(6)\end{array}$ & $1.315(7)$ & $2.900(2)$ & $116.4(3)$ & this work \\
\hline$\left[\mathrm{Mo}\left(\mathrm{Et}_{2} \mathrm{NCS}_{2}\right)(\mathrm{CO})(\mathrm{NO})(\mathrm{dppe})\right]$ & $\begin{array}{l}2.549(1) \\
2.535(1)\end{array}$ & $\begin{array}{l}1.709(5) \\
1.714(5)\end{array}$ & $1.350(6)$ & $2.891(2)$ & $115.2(3)$ & this work \\
\hline$\left[\mathrm{Mo}\left(\mathrm{Et}_{2} \mathrm{NCS}_{2}\right)(\mathrm{CO})(\mathrm{NO})\left(\mathrm{PPh}_{3}\right)_{2}\right]$ & $\begin{array}{l}2.565(2) \\
2.576(1)\end{array}$ & $\begin{array}{l}1.707(5) \\
1.710(5)\end{array}$ & $1.311(7)$ & $2.901(2)$ & $116.2(3)$ & this work \\
\hline$\left[\mathrm{Mo}\left(\mathrm{Et}_{2} \mathrm{NCS}_{2}\right)(\mathrm{CO})(\mathrm{NO})\left(\mathrm{P}\{\mathrm{OMe}\}_{3}\right)_{2}\right]$ & $\begin{array}{l}2.565(4) \\
2.559(4)\end{array}$ & $\begin{array}{l}1.68(1) \\
1.69(2)\end{array}$ & $1.36(2)$ & $2.888(5)$ & $117.6(8)$ & this work \\
\hline
\end{tabular}

${ }^{a} \mathrm{C}_{4} \mathrm{H}_{8} \mathrm{NCS}_{2}{ }^{-}=$pyrrolidine-1-carbodithioato.

${ }^{\mathrm{D}} \mathrm{C}_{5} \mathrm{H}_{10} \mathrm{NCS}_{2}{ }^{-}=$piperidine-1-carbodithioato.

'The structure contains a crystallographically imposed two-fold axis, through the Mo atom and the $\mathrm{C}-\mathrm{N}$ bond.

3 ), indicating a quasi-octahedral environment. The observed bond angles, $\angle \mathrm{M}-\mathrm{N}-\mathrm{O}$, which lie within the range $175.1-177.7^{\circ}$, show that the nitrosyl group can be considered linear ' $\mathrm{NO}^{+}$' in these $\mathrm{Mo}^{\circ}$ compounds [10].

The S $\cdots$ S 'bite' distances of the diethyldithiocarbamato ligands between 2.888 and $2.901 \AA$ (Table 4) are typical for bidentate geometry [13]. This is also compatible with the expectation of the ligand as a fourelectron donor, based on a simple electron counting rule (e.g., 18-electron rule). This conclusion is further supported by the two nearly identical $\mathrm{C}-\mathrm{S}$ bond lengths in each ligand. However, the angles, $\angle \mathrm{S}-\mathrm{C}-\mathrm{S}$, which lie in the range $114.5-117.6^{\circ}$, contain the value of $115.7^{\circ}$ observed for the monodentate diethyldithiocarbamato ligand in $\left[\mathrm{Pt}\left(\eta^{1}-\mathrm{Et}_{2} \mathrm{NCS}_{2}\right)\left(\left\{\mathrm{PhP}\left(\mathrm{CH}_{2} \mathrm{CH}_{2} \mathrm{PPh}_{2}\right)_{2}\right\}\right] \mathrm{BPh}_{4}\right.$ [13] so that this angle cannot be used as a criterion for judging a mono- or bidentate coordination mode for the ligand.

Although the $S \cdots S$ values of $1,3,4,6,7,8$ and 10 are similar to that of $2.908(2) \AA$ in [PPN][Mo$\left.\left(\mathrm{C}_{4} \mathrm{H}_{8} \mathrm{NCS}_{2}\right)(\mathrm{CO})_{4}\right](11), 2.892(6) \AA$ in [PPN][Mo$\left.\left(\mathrm{C}_{5} \mathrm{H}_{10} \mathrm{NCS}_{2}\right)(\mathrm{CO})_{4}\right] \quad(12) \quad\left(\mathrm{C}_{4} \mathrm{H}_{8} \mathrm{NCS}_{2}{ }^{-}=\right.$pyrroline-1carbodithioato; $\quad \mathrm{C}_{5} \mathrm{H}_{10} \mathrm{NCS}_{2}{ }^{-}=$piperidine-1-carbodithioato) [12], and 2.90(1) $\AA$ in [PPN] $\left[\mathrm{Mo}\left(\mathrm{Et}_{2} \mathrm{NCS}_{2}\right)\right.$ $(\mathrm{CO})_{4}$ ] (13) [11], comparison of the Mo-S distances in 1-13 (Table 4) reveals two types with the longer values $(2.597-2.631 \AA)$ for the tetracarbonyl complexes, 11-13, and the shorter values (2.518-2.576 $\AA$ ) for $\left[\mathrm{Mo}\left(\mathrm{Et}_{2} \mathrm{NCS}_{2}\right)(\mathrm{NO})(\mathrm{CO}) \mathrm{L}_{2}\right]^{m-}$. Apparently, if relative contribution of the canonical forms I, II and III is used to represent the $\sigma$-donicity of a dialkyldithiocarbamato ligand, as depicted below, the presence of a stronger $\pi$-acid, NO, in the structures can permit a greater contribution of III of the diethyldithiocarbamato

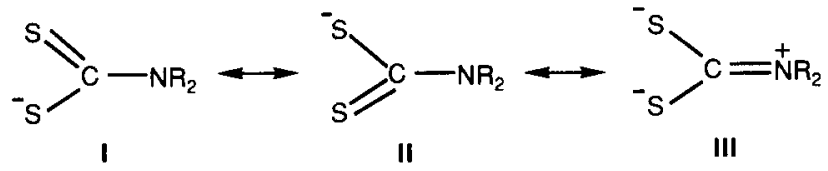

ligand. However, as reflected in the similar $\mathrm{C}-\mathrm{N}$ distances (Table 4), one should also be cautious in that some modest difference, as large as $0.03 \AA[11,14]$, in the singly bonded distances may not indicate chemical effects but may be caused by the crystal packing effects.

\section{Supplementary material}

Tables of complete bond lengths and angles, anisotropic displacement parameters, hydrogen coordinates, observed and calculated structure factors can be obtained from the authors upon request.

\section{Acknowledgement}

The authors thank the National Science Council of the Republic of China for financial support of this research (Contract No. NSC83-0208-M006-35). 


\section{References}

[1] K.-B. Shiu, K.-S. Liou, S.-L. Wang, C.P. Cheng, B.R. Fang and W.-J. Vong, Organometallics, 8 (1989) 1219.

[2] K.-B. Shiu, C.-J. Chang, Y. Wang and M.-C. Cheng, J. Organomet. Chem., 406 (1991) 363.

[3] S.-T. Lin, M.Sc. Thesis, National Cheng Kung University, Tainan, Taiwan, 1993.

[4] E.J. Gabe, Y.L. Page, P.S. White and F.L. Lee, Acta Crystallogr., Sect. $A, 43$ (1987) S294.

[5] International Tables for X-Ray Crystallography, Vol. 4, Kynoch, Birmingham, UK, 1974.

[6] L.-K. Liu, J.-T. Lin and D. Fang, Inorg. Chim. Acta, 161 (1989) 239.

[7] E.L. Eliel, Stereochemistry of Carbon Compounds, McGraw-Hill, New York, 1962, p. 207.
[8] J.H. Letcher and J.R. Van Wazer, J. Chem. Phys., 44 (1966) 815.

[9] B.M. Trost and M. Lautens, J. Am. Chem. Soc., 104 (1982) 5543.

[10] J.P. Collman, L.S. Hegedus, J.R. Norton and R.G. Finks, Principles and Applications of Organotransition Metal Chemistry, University Science Books, Mill Valley, CA, 1987, p. 192.

[11] K.-B. Shiu, S.-M. Peng, Y. Wang and M.-C. Cheng, Proc. 2nd ROC-Japan Joint Seminar Crystallography, Taipei Taiwan, 1992, p. 113.

[12] K.-B. Shiu, S.-M. Peng, M.-C. Cheng, S.-L. Wang and F.-L. Liao, J. Organomet. Chem., 461 (1993) 111.

[13] R. Colton, M.F. Mackay and V. Tedesco, Inorg. Chim. Acta, 207 (1993) 227.

[14] A.L. Rheingold and J.R. Harper, J. Organomet. Chem., 403 (1991) 335. 